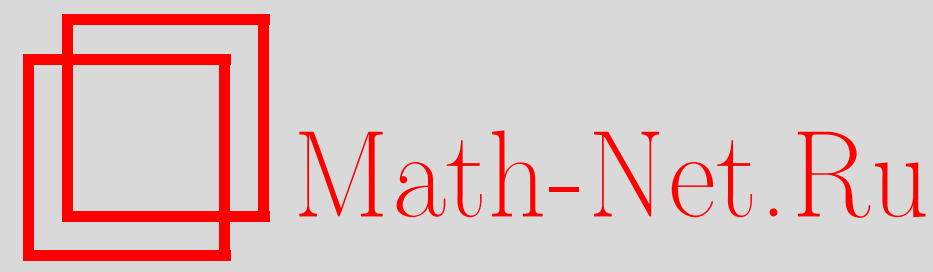

3. Ю. Фазуллин, Х. Х. Муртазин, Регуляризованный след двумерного гармонического осциллятора, Матем. сб., 2001, том 192, номер 5, 87-124

DOI: https://doi.org/10.4213/sm566

Использование Общероссийского математического портала Math-Net.Ru подразумевает, что вы прочитали и согласны с пользовательским соглашением

http://www . mathnet.ru/rus/agreement

Параметры загрузки:

IP: 54.162 .85 .209

26 апреля 2023 г., $16: 46: 50$ 


\author{
З. Ю. Фазуллин, Х.Х. Муртазин
}

\title{
Регуляризованный след двумерного гармонического осциллятора
}

\footnotetext{
В статье исследован спектр финитного возмущения двумерного гармонического осциллятора и получена классическая формула первого регуляризованного следа.

Библиограффия: 12 названий.
}

\section{Введение}

Классической формулой регуляризованного следа порядка $m$ оператора $H$ называется соотношение вида

$$
\sum_{k}\left(\mu_{k}^{m}-A_{k}(m)\right)=B(m)
$$

где $\mu_{k}$ - собственные значения оператора $H, m \in \mathbb{N}$, а $A_{k}(m)$ и $B(m)$ - явно вычисляемые через характеристики оператора выражения.

Впервые формула такого вида для оператора Штурма-Лиувилля на конечном отрезке была получена в работе [1] И.М. Гельфандом и Б. М. Левитаном. Наиболее общие результаты для обыкновенных дифференциальных операторов получены В.Б. Лидским и В.А. Садовничим в работе [2], где установлено, что доказательство формул вида (0.1) для широкого класса краевых задач, порожденных обыкновенными дифференциальными выражениями на конечном отрезке, сводится к изучению регуляризованных сумм корней целых функций с определенной асимптотической структурой.

Ситуация значительно усложняется при рассмотрении задач, порожденных дифференциальными операторами с частными производными. Это обусловлено, прежде всего, сложной структурой спектра.

Большим продвижением стала работа В.А. Садовничего и В.В. Дубровского [3], в которой доказана формула типа (0.1) для оператора Лапласа-Бельтрами, возмушенного гладким нечетным потенциалом на двумерной единичной сфере. В работе [4] В.Е. Подольским получены формулы типа (0.1) для оператора Лапласа-Бельтрами с потенциалом на компактных симметрических пространствах ранга 1.

В настоящей работе изучается оператор $H=H_{0}+V$ в $L^{2}\left(\mathbb{R}^{2}\right)$, где $H_{0}=-\Delta+x^{2}$, $x^{2}=x_{1}^{2}+x_{2}^{2}, V$ - оператор умножения на ограниченную измеримую финитную вешественную функцию.

(C) З. Ю. ФАЗУЛлИН, Х.Х. МУРтАЗИН 2001 
Исследован спектр оператора $H$ и получена классическая формула типа (0.1) регуляризованного следа для собственных чисел оператора $H$. Отметим, что в случае радиального финитного $V$, обращающегося в нуль в окрестности нуля, равенство нулю регуляризованной суммы для собственных чисел оператора $H$ анонсировано в [5]. Однако в силу радиальности возмушения этот случай мало отличается от одномерного гармонического осциллятора, кроме того, полученное соотношение для собственных значений не является формулой типа (0.1).

Авторы выражают искреннюю благодарность В. А. Садовничему за полезные обсуждения.

Сформулируем вспомогательные утверждения.

Спектр оператора $H_{0}$ хорошо известен и состоит из собственных значений $\lambda_{n}=$ $2 n+2, n \geqslant 0$. Соответствующие проекторы на собственные подпространства $($ размерности $n+1)$ имеют вид

$$
P_{n} h=\sum_{l=0}^{n}\left(h, \varphi_{l}^{(n)}\right) \varphi_{l}^{(n)}
$$

где $(\cdot, \cdot)$ - скалярное произведение в $L^{2}\left(\mathbb{R}^{2}\right), \varphi_{l}^{(n)}(x)=f_{l}\left(x_{1}\right) f_{n-l}\left(x_{2}\right), f_{l}(t)-$ нормированная собственная функция одномерного гармонического осциллятора, соответствуюшая собственному числу $2 l+1(l \geqslant 0)$.

Хорошо известно, что

$$
f_{l}(t)=\left(2^{l} l ! \sqrt{\pi}\right)^{-1 / 2} e^{-t^{2} / 2} H_{l}(t)
$$

где $H_{l}(t)$ - многочлены Эрмита, причем на любом компакте $K \subset \mathbb{R}$ имеет место глобальная оценка [6; гл. $2, \S 7$, п. 2 , формула $(21)]$

$$
\left|f_{l}(t)\right| \leqslant \frac{c_{0}}{\sqrt[4]{2 l+1}}
$$

$c_{0}>0$ зависит только от $K$.

Из асимптотической формулы для многочленов $H_{n}(t)[7 ;$ гл. 8 , теорема 8.22.6] и формулы Стирлинга [6; дополнение A, формула (22)] следует, что при $l \gg 1, t \in K$ $(K-$ компакт в $\mathbb{R})$

$$
\begin{aligned}
f_{l}(t)= & \alpha_{l}\left\{\cos \left[\sqrt{2 l+1} t-\frac{l \pi}{2}\right]\left[u_{0}(t)-\frac{u_{2}(t)}{4(2 l+1)}+\frac{u_{4}(t)}{16(2 l+1)^{2}}+O\left(\frac{1}{l^{3}}\right)\right]\right. \\
& \left.+\sin \left[\sqrt{2 l+1} t-\frac{l \pi}{2}\right]\left[\frac{u_{1}(t)}{2(2 l+1)^{1 / 2}}-\frac{u_{3}(t)}{8(2 l+1)^{3 / 2}}+O\left(\frac{1}{l^{5 / 2}}\right)\right]\right\},
\end{aligned}
$$

где

$$
\begin{aligned}
u_{0}(t) & =1, \quad u_{l}(t)=\int_{0}^{t} L u_{l-1}(\tau) d \tau, \quad L=-\frac{d^{2}}{d t^{2}}+t^{2} \\
\alpha_{l} & =\sqrt{\frac{2}{\pi}} \frac{1}{\sqrt[4]{2 l+1}}\left\{1-\frac{1}{32} \frac{1}{(2 l+1)^{2}}+O\left(\frac{1}{l^{3}}\right)\right\} .
\end{aligned}
$$


В дальнейших исследованиях мы часто будем пользоваться интегральными приближениями сумм. А именно справедлива

Лемма 0.1. Пусть $\left\{\lambda_{k}\right\}, k=M_{0}, M_{0}+1, \ldots$, - конечное или бесконечное $\left(\lambda_{\infty}=\infty\right)$ подмножество натуральных чисел, $\lambda_{k+1}-\lambda_{k}=c_{0}>0, k=$ $M_{0}, M_{0}+1, \ldots, u g \in C^{3}(X), X=\left[\lambda_{M_{0}}, \lambda_{M+1}\right]$ npu $\lambda_{M+1}<\infty\left(X=\left[\lambda_{M_{0}}, \infty\right)\right.$ в бесконечном случае).

Если последовательность $g\left(\lambda_{k}\right)$ суммируема, то справедлива формула

$$
\begin{aligned}
\sum_{k=M_{0}}^{M} g\left(\lambda_{k}\right)=\frac{1}{c_{0}} & \int_{\lambda_{M_{0}}}^{\lambda_{M+1}} g(t) d t-\frac{1}{2}\left[g\left(\lambda_{M+1}\right)-g\left(\lambda_{M_{0}}\right)\right] \\
& +\frac{c_{0}}{12}\left[g^{\prime}\left(\lambda_{M+1}\right)-g^{\prime}\left(\lambda_{M_{0}}\right)\right]+\Gamma\left(M_{0}, M\right)
\end{aligned}
$$

əде

$$
\Gamma\left(M_{0}, M\right)=\sum_{k=M_{0}}^{M} \int_{\lambda_{k}}^{\lambda_{k+1}}\left[\frac{\left(t-\lambda_{k+1}\right)^{3}}{6 c_{0}}+\frac{\left(t-\lambda_{k+1}\right)^{2}}{4}+\frac{c_{0}}{12}\left(t-\lambda_{k+1}\right)\right] g^{\prime \prime \prime}(t) d t
$$

ДокАЗАТЕЛьство. Из соотношений (0.6) и (0.7), так как $c_{0}=\lambda_{k+1}-\lambda_{k}$, вытекает, что

$$
\begin{aligned}
c_{0} g\left(\lambda_{k}\right)= & \int_{\lambda_{k}}^{\lambda_{k+1}} g(t) d t-\frac{c_{0}}{2} \int_{\lambda_{k}}^{\lambda_{k+1}} g^{\prime}(t) d t+\frac{c_{0}^{2}}{12} \int_{\lambda_{k}}^{\lambda_{k+1}} g^{\prime \prime}(t) d t \\
& +\int_{\lambda_{k}}^{\lambda_{k+1}}\left[\frac{\left(t-\lambda_{k+1}\right)^{3}}{6}+\frac{c_{0}\left(t-\lambda_{k+1}\right)^{2}}{4}+\frac{c_{0}^{2}\left(t-\lambda_{k+1}\right)}{12}\right] g^{\prime \prime \prime}(t) d t .
\end{aligned}
$$

Лемма 0.1 будет доказана, если убедимся, что (0.8) есть тождество. С этой целью последнее слагаемое в (0.8) три раза проинтегрируем по частям и получим

$$
\begin{aligned}
& \int_{\lambda_{k}}^{\lambda_{k+1}}\left[\frac{\left(t-\lambda_{k+1}\right)^{3}}{6}+\frac{c_{0}\left(t-\lambda_{k+1}\right)^{2}}{4}+\frac{c_{0}^{2}\left(t-\lambda_{k+1}\right)}{12}\right] g^{\prime \prime \prime}(t) d t \\
& \quad=-\frac{c_{0}^{2}}{12} \int_{\lambda_{k}}^{\lambda_{k+1}} g^{\prime \prime}(t) d t+\frac{c_{0}}{2} \int_{\lambda_{k}}^{\lambda_{k+1}} g^{\prime}(t) d t-\int_{\lambda_{k}}^{\lambda_{k+1}} g(t) d t+c_{0} g\left(\lambda_{k}\right)
\end{aligned}
$$

Поставляя полученное равенство в (0.8), получаем требуемое тождество. Лемма 0.1 доказана.

В частности, если $g \in C^{2}(X)$, имеем

$$
\begin{aligned}
\sum_{k=M_{0}}^{M} g\left(\lambda_{k}\right)= & \frac{1}{c_{0}} \int_{\lambda_{M_{0}}}^{\lambda_{M+1}} g(t) d t-\frac{1}{2}\left[g\left(\lambda_{M+1}\right)-g\left(\lambda_{M_{0}}\right)\right] \\
& -\sum_{k=M_{0}}^{M} \int_{\lambda_{k}}^{\lambda_{k+1}}\left[\frac{\left(t-\lambda_{k+1}\right)^{2}}{2 c_{0}}+\frac{t-\lambda_{k+1}}{2}\right] g^{\prime \prime}(t) d t
\end{aligned}
$$




\section{§1. Локализация спектра}

Введем оператор $P_{k} h=\sum_{s=0}^{k}\left(h, \varphi_{s}^{(k)}\right) \varphi_{s}^{(k)}($ см. $(0.2))$ и рассмотрим спектральную задачу

$$
H_{0} u+V u=z u .
$$

Пусть $P_{k} u=u_{k}$, тогда $u=\sum_{m=0}^{\infty} u_{m}$ - ортогональное разложение $u$. Для последовательности $u_{k}$ справедлива система уравнений

$$
2(k+1) u_{k}+\sum_{m=0}^{\infty} V_{k m} u_{m}=z u_{k}, \quad k=0,1, \ldots
$$

где $V_{k m}=P_{k} V P_{m}$, причем

$$
\|u\|^{2}=\sum_{m=0}^{\infty}\left\|u_{m}\right\|^{2}
$$

и уравнения (1.1) и (1.2) эквивалентны. С целью исследования системы (1.2) вначале покажем, что справедлива

Лемма 1.1. Пусть $V(x)-$ финитная ограниченная функиия $и \operatorname{supp} V \in$ $[a, b] \times[c, d]$, тогда при $k \gg 1$

$$
\left\|P_{k} V\right\| \leqslant \frac{M\|V\|_{\infty}}{\sqrt[4]{k+1}}
$$

где $M$ зависит только от $\operatorname{supp} V,\|V\|_{\infty}=\sup _{x \in \operatorname{supp} V}|V(x)|$.

ДокАЗАТЕЛЬСТво. Пусть $h \in L_{2}\left(\mathbb{R}^{2}\right)$, тогда имеем

$$
\left\|P_{k} V h\right\|^{2}=\sum_{s=0}^{k}\left|\left(V h, \varphi_{s}^{(k)}\right)\right|^{2} .
$$

Эту сумму разобьем на две части:

$$
\left\|P_{k} V h\right\|^{2}=\sum_{s=0}^{\left[\frac{k}{2}\right]}\left|\left(V h, \varphi_{s}^{(k)}\right)\right|^{2}+\sum_{s=\left[\frac{k}{2}\right]+1}^{k}\left|\left(V h, \varphi_{s}^{(k)}\right)\right|^{2}
$$

где $\left[\frac{k}{2}\right]$ - целая часть числа $\frac{k}{2}$. Так как

$$
\left(V h, \varphi_{s}^{(k)}\right)=\int_{a}^{b} \int_{c}^{d} V\left(x_{1}, x_{2}\right) h\left(x_{1}, x_{2}\right) f_{s}\left(x_{1}\right) f_{k-s}\left(x_{2}\right) d x_{1} d x_{2},
$$


используя неравенство Коши-Буняковского по переменной $x_{2}$ и неравенство Бесселя по системе $\left\{f_{l}\right\}_{l=0}^{\infty}$, а также неравенство (0.4), для первой суммы в (1.4) будем иметь оценку

$$
\begin{aligned}
\sum_{s=0}^{\left[\frac{k}{2}\right]}\left|\left(V h, \varphi_{s}^{(k)}\right)\right|^{2} & \leqslant c_{0}^{2}([c, d])(d-c) \sum_{s=0}^{\left[\frac{k}{2}\right]} \frac{\int_{c}^{d}\left|\int_{a}^{b} V(x) h(x) f_{s}\left(x_{1}\right) d x_{1}\right|^{2} d x_{2}}{\sqrt{2(k-s)+1}} \\
& \leqslant \frac{c_{0}^{2}([c, d])(d-c)}{\sqrt{k+1}} \int_{c}^{d} \int_{a}^{b}|V(x) h(x)|^{2} d x_{1} d x_{2} \\
& \leqslant \frac{c_{0}^{2}([c, d])(d-c)}{\sqrt{k+1}}\|V\|_{\infty}^{2}\|h\|^{2}
\end{aligned}
$$

Аналогично находим, что

$$
\sum_{s=\left[\frac{k}{2}\right]+1}^{k}\left|\left(V h, \varphi_{s}^{(k)}\right)\right|^{2} \leqslant \frac{c_{0}^{2}([c, d])(d-c)}{\sqrt{k+1}}\|V\|_{\infty}^{2}\|h\|^{2} .
$$

Из этих неравенств и равенства (1.4) следует лемма 1.1.

Основным результатом этого параграфа является

ТЕОРема 1.1. Пусть $n \gg 1$, тогда собственные значения $z$ оператора $H$, лежащие в окрестности точки $2(n+1)$, удовлетворяют неравенству

$$
|2(n+1)-z| \leqslant M^{2}\|V\|_{\infty}\left(\frac{1}{\sqrt{n}}+O\left(\frac{\ln n}{n}\right)\right) .
$$

ДокаЗАТЕЛЬСтво. Пусть собственное значение $z$ оператора $H$ не принадлежит спектру оператора $H_{0}$. Поскольку $u_{k}=\frac{P_{k} V u}{z-2(k+1)}$, то по лемме $1.1\left\|u_{k}\right\| \leqslant$ $\frac{M\|V\|_{\infty}\|u\|}{|z-2(k+1)| \sqrt[4]{k+1}}$, отсюда следует, что $\|u\|_{1}=\sum_{k=0}^{\infty}\left\|u_{k}\right\|<\infty$. Поэтому для анализа системы (1.2) можно использовать норму $\|u\|_{1}$. Согласно (1.2)

$$
u_{k}=\frac{1}{z-2(k+1)} \sum_{m=0}^{\infty} P_{k} V P_{m} u_{m}
$$

Пусть $\theta$ - оператор умножения на характеристическую функцию $\operatorname{supp} V$, тогда по лемме 1.1

$$
\left\|P_{k} V P_{m}\right\|=\left\|P_{k} \theta V \theta P_{m}\right\| \leqslant \frac{M\|V\|_{\infty}}{\sqrt[4]{k+1}}\left\|\theta P_{m}\right\| \leqslant \frac{M^{2}\|V\|_{\infty}}{\sqrt[4]{k+1} \sqrt[4]{m+1}},
$$

следовательно,

$$
\left\|u_{k}\right\| \leqslant \frac{M^{2}\|V\|_{\infty}}{|z-2(k+1)| \sqrt[4]{k+1}} \sum_{m=0}^{\infty} \frac{\left\|u_{m}\right\|}{\sqrt[4]{m+1}}=\frac{M^{2}\|V\|_{\infty}^{2}\|u\|_{2}}{|z-2(k+1)| \sqrt[4]{k+1}},
$$


где

$$
\|u\|_{2}=\sum_{m=0}^{\infty} \frac{\left\|u_{m}\right\|}{\sqrt[4]{m+1}}
$$

Отсюда следует оценка

$$
\|u\|_{2}=\sum_{k=0}^{\infty} \frac{\left\|u_{k}\right\|}{\sqrt[4]{k+1}} \leqslant M^{2}\|V\|_{\infty}\|u\|_{2} \sum_{k=0}^{\infty} \frac{1}{|z-2(k+1)| \sqrt{k+1}}
$$

Полученное неравенство позволяет доказать теорему 1.1 путем элементарных рассуждений. Пусть $n \gg 1$ и $z \in[2 n+1,2 n+3], z \neq 2 n+2$. Легко убедиться, что

$$
\sum_{k=0}^{n-1} \frac{1}{\sqrt{k+1}|z-2(k+1)|}=O\left(\frac{\ln n}{\sqrt{n}}\right), \quad \sum_{k=n+1}^{\infty} \frac{1}{\sqrt{k+1}|z-2(k+1)|}=O\left(\frac{\ln n}{\sqrt{n}}\right)
$$

Следовательно, из (1.5) имеем неравенство

$$
\|u\|_{2} \leqslant M^{2}\|V\|_{\infty}\|u\|_{2}\left(\frac{1}{\sqrt{n+1}|z-2(n+1)|}+O\left(\frac{\ln n}{\sqrt{n}}\right)\right) .
$$

Допустим, что $\sqrt{n+1}|z-2(n+1)| \rightarrow \infty$ при $n \rightarrow \infty$, тогда из неравенства (1.6) имеем, что $\|u\|_{2}=0$ и $u \equiv 0$, т.е. $z$ не является собственньм значением оператоpa $H$. Следовательно, $|z-2(n+1)|=O\left(n^{-1 / 2}\right)$. Отсюда и из неравенства (1.6) вытекает утверждение теоремы 1.1.

\section{§2. Сумма третьей поправки теории возмущений}

Пусть $R_{0}(z)=\left(H_{0}-z\right)^{-1}, R(z)=(H-z)^{-1}$, тогда имеем

$$
R(z)=R_{0}(z)-R_{0}(z) V R_{0}(z)+\left(R_{0}(z) V\right)^{2} R_{0}(z)-\left(R_{0}(z) V\right)^{2} R(z) V R_{0}(z)
$$

Обозначим через $\mu_{k}^{(n)}, k=\overline{0, n}$, собственные значения оператора $H$, лежащие в окрестности $\lambda_{n}=2(n+1)$ - собственного числа оператора $H_{0}$. Тогда справедлива

ЛЕмма 2.1. При $n \gg 1$

$$
2(n+1)^{2}+\operatorname{sp} P_{n} V-\sum_{k=0}^{n} \mu_{k}^{(n)}=\alpha_{n}+\beta_{n}
$$

где $\mathrm{sp}$ - след ядерного оператора,

$$
\begin{aligned}
& \alpha_{n}=\operatorname{sp} P_{n} V B_{n} V P_{n}, \quad B_{n}=\sum_{m \neq n}\left(\lambda_{m}-\lambda_{n}\right)^{-1} P_{m} \\
& \beta_{n}=\operatorname{sp}\left(-\frac{1}{2 \pi i} \oint_{\left|\lambda_{n}-z\right|=1} z\left(R_{0}(z) V\right)^{2} R(z) V R_{0}(z) d z\right) .
\end{aligned}
$$


ДоКАЗАТЕЛЬСтво. В силу компактности $R_{0}(z)$ имеем, что

$$
R_{0}(z)=\frac{P_{n}}{\lambda_{n}-z}+R_{1}(z)
$$

где $R_{1}(z)=\sum_{m \neq n}\left(\lambda_{m}-z\right)^{-1} P_{m}$ регулярна в окрестности $\lambda_{n}$, причем $P_{m} P_{n}=$ $\delta_{m n} P_{m}$, поэтому

$$
P_{n} R_{1}(z)=R_{1}(z) P_{n}=0 .
$$

Далее из $[8 ;$ гл. IV,$\S 3.4$, теорема 3.16$]$ и теоремы 1.1 в силу компактности $R(z)$ следует, что

$$
R(z)=\sum_{i=0}^{n} \frac{Q_{i}}{\mu_{i}^{(n)}-z}+G(z)
$$

где $Q_{i}$ - проектор на собственное пространство, соответствующее $\mu_{i}^{(n)}, G(z)$ регулярна в круге $\left|\lambda_{n}-z\right| \leqslant 1$. Теперь для доказательства формулы $(2.2)$ к обеим частям равенства (2.1) применим оператор

$$
\operatorname{sp}\left(\frac{1}{2 \pi i} \oint_{\left|\lambda_{n}-z\right|=1} z(\cdot) d z\right) \text {. }
$$

Тогда из соотношений $(2.5)$ и (2.7), используя теорему о вычетах, получаем

$$
\begin{aligned}
\operatorname{sp}\left(\frac{1}{2 \pi i} \oint_{\left|\lambda_{n}-z\right|=1} z\left(R(z)-R_{0}(z)\right) d z\right) & =\operatorname{sp}\left(\lambda_{n} P_{n}-\sum_{i=0}^{n} \mu_{i}^{(n)} Q_{i}\right) \\
& =2(n+1)^{2}-\sum_{i=0}^{n} \mu_{i}^{(n)}
\end{aligned}
$$

Далее, так как в силу (2.6) $\operatorname{sp}\left(P_{n} V R_{1}\left(\lambda_{n}\right)\right)=\operatorname{sp}\left(R_{1}\left(\lambda_{n}\right) V P_{n}\right)=0$, имеем

$$
\begin{aligned}
& \operatorname{sp}\left(\frac{1}{2 \pi i} \oint_{\left|\lambda_{n}-z\right|=1} z R_{0}(z) V R_{0}(z) d z\right) \\
& \quad=\operatorname{sp}\left[P_{n} V P_{n}-\lambda_{n}\left(P_{n} V R_{1}\left(\lambda_{n}\right)+R_{1}\left(\lambda_{n}\right) V P_{n}\right)\right]=\operatorname{sp} P_{n} V .
\end{aligned}
$$

Для третьего слагаемого в правой части равенства (2.1) получим

$$
\alpha_{n}=\operatorname{sp}\left(\frac{1}{2 \pi i} \oint_{\left|\lambda_{n}-z\right|=1} z\left(R_{0}(z) V\right)^{2} R_{0}(z) d z\right)=\operatorname{sp}\left[P_{n} V R_{1}\left(\lambda_{n}\right) V P_{n}\right] .
$$

Для доказательства (2.10) подставим (2.5) в левую часть этого равенства и вычислим вычеты в точке $z=\lambda_{n}$. Учитывая равенство (2.6), получаем, что

$$
\alpha_{n}=\operatorname{sp}\left(P_{n} V R_{1}\left(\lambda_{n}\right) V P_{n}\right)+\lambda_{n}\left[\operatorname{sp}\left(P_{n} V R_{1}^{\prime}\left(\lambda_{n}\right) V P_{n}\right)-\operatorname{sp}\left(P_{n} V R_{1}^{2}\left(\lambda_{n}\right) V P_{n}\right)\right] .
$$

Отсюда, так как $R_{1}^{\prime}\left(\lambda_{n}\right)=R_{1}^{2}\left(\lambda_{n}\right)$, следует (2.10). Следовательно, замечая, что $R_{1}\left(\lambda_{n}\right)=\sum_{m \neq n}\left(\lambda_{m}-\lambda_{n}\right)^{-1} P_{m}$, из равенств $(2.8)-(2.10)$ получаем доказательство леммы 2.1 .

Формула регуляризованного следа непосредственно вытекает из равенства (2.2), а именно мы показываем, что последовательности $\alpha_{n}$ и $\beta_{n}$ суммируемы. Справедлива 
ТЕОРема 2.1. Последовательность $\beta_{k}$ абсолютно суммируема $и$

$$
\sum_{k=0}^{\infty} \beta_{k}=0
$$

ДокАЗАТЕльСтво. Пусть $\Phi(z)=\left(R_{0}(z) V\right)^{2} R(z) V R_{0}(z)$. Так как $\lambda_{n}=2 n+2$, оператор $\Phi(z)$ ядерный, следовательно,

$$
\beta_{n}=-\operatorname{sp}\left(\frac{1}{2 \pi i} \oint_{\left|\lambda_{n}-z\right|=1} z \Phi(z) d z\right)=-\frac{1}{2 \pi i} \oint_{\left|\lambda_{n}-z\right|=1} z \operatorname{sp} \Phi(z) d z
$$

Отсюда в силу теоремы 1.1 следует, что

$$
\sigma_{n}=\sum_{k=0}^{n} \beta_{k}=-\frac{1}{2 \pi i} \oint_{\Gamma_{n}} z \operatorname{sp} \Phi(z) d z
$$

где $\Gamma_{n}=\Pi_{n}(\varphi) \cup \gamma_{n}, \Pi_{n}(\varphi)=\left\{z: z=(2 n+3) \sqrt{2} e^{i \varphi}, \pi / 4 \leqslant \varphi \leqslant 7 \pi / 4\right\}$, $\gamma_{n}=\{z: z=2 n+3+i t,|t| \leqslant 2 n+3\}$. Теорема 2.1 будет доказана, если убедимся, что

$$
\begin{aligned}
& \sigma_{n}^{(1)}=-\frac{1}{2 \pi} \int_{-2 n-3}^{2 n+3} t \operatorname{sp} \Phi(2 n+3+i t) d t \rightarrow 0, \quad n \rightarrow \infty, \\
& \sigma_{n}^{(2)}=-\frac{1}{2 \pi i} \int_{-2 n-3}^{2 n+3}(2 n+3) \operatorname{sp} \Phi(2 n+3+i t) d t \rightarrow 0, \quad n \rightarrow \infty, \\
& \sigma_{n}^{(3)}=-\frac{1}{2 \pi i} \int_{\Pi_{n}(\varphi)} z \operatorname{sp} \Phi(z) d z \rightarrow 0, \quad n \rightarrow \infty .
\end{aligned}
$$

Перейдем к доказательству соотношений (2.11)-(2.13). Поскольку

$$
\operatorname{sp} \Phi(2 n+3+i t)=\sum_{k=0}^{\infty} \sum_{s=0}^{k} \frac{\left(V R_{0}(2 n+3+i t) V R(2 n+3+i t) V \varphi_{s}^{(k)}, \varphi_{s}^{(k)}\right)}{(2(k-n)-1-i t)^{2}},
$$

для оценки $|\operatorname{sp} \Phi(2 n+3+i t)|$ докажем, что имеет место

Лемма 2.2. Пусть $h \in L_{2}\left(\mathbb{R}^{2}\right)$. Тогда при $n \gg 1$

$$
\begin{aligned}
\left\|R_{0}(2 n+3+i t) \theta h\right\| \leqslant \mathrm{const} \cdot n^{-\alpha / 2}(1+|t|)^{-1+\alpha}\|h\|, & 0<\alpha<1, \\
\|R(2 n+3+i t) \theta h\| \leqslant \mathrm{const} \cdot n^{-\alpha / 2}(1+|t|)^{-1+\alpha}\|h\|, & 0<\alpha<1,
\end{aligned}
$$

где $\theta(x)$ - характеристическая функиия $\operatorname{supp} V$. 
ДоКАЗАТЕЛЬСтво. Пусть $h \in L^{2}\left(\mathbb{R}^{2}\right)$, тогда

$$
u=R_{0}(2 n+3+i t) \theta h=\sum_{m=0}^{\infty} \frac{P_{m} \theta h}{2(m-n)-1-i t},
$$

откуда следует, что

$$
\|u\|^{2}=\sum_{m=0}^{\infty} \frac{\left\|P_{m} \theta h\right\|^{2}}{[2(m-n)-1]^{2}+t^{2}} .
$$

Далее, пусть $0<\sigma<1$, тогда в силу неравенства Бесселя

$$
\sum_{m=0}^{n-\left[n^{\sigma}\right]} \frac{\left\|P_{m} \theta h\right\|^{2}}{(2(m-n)-1)^{2}+t^{2}} \leqslant \frac{\text { const } \cdot\|\theta h\|^{2}}{n^{2 \sigma}+t^{2}} \leqslant \frac{\text { const } \cdot\|h\|^{2}}{n^{2 \sigma \alpha}(1+|t|)^{2(1-\alpha)}} .
$$

Мы здесь также воспользовались неравенством

$$
a^{2}+b^{2} \geqslant \text { const } \cdot a^{2 \alpha} b^{2(1-\alpha)}, \quad 0<\alpha<1 \text {. }
$$

Аналогично

$$
\sum_{m=n+\left[n^{\sigma}\right]}^{\infty} \frac{\left\|P_{m} \theta h\right\|^{2}}{(2(m-n)-1)^{2}+t^{2}} \leqslant \frac{\text { const } \cdot\|h\|^{2}}{n^{2 \sigma \alpha}(1+|t|)^{2(1-\alpha)}}
$$

Теперь оценим промежуточное слагаемое суммы (2.17). Из леммы 1.1 и неравенства (2.19) получим

$$
\sum_{m=n-\left[n^{\sigma}\right]+1}^{n+\left[n^{\sigma}\right]-1} \frac{\left\|P_{m} \theta h\right\|^{2}}{(2(m-n)-1)^{2}+t^{2}} \leqslant \frac{\text { const } \cdot\|h\|^{2}}{n^{(2 \alpha-1) \sigma+1 / 2}(1+|t|)^{2(1-\alpha)}} .
$$

Таким образом, из (2.18) и (2.20), (2.21) заключаем, что

$$
\|u\|^{2} \leqslant \frac{\text { const } \cdot\|h\|^{2}}{n^{2 \alpha \sigma}(1+|t|)^{2(1-\alpha)}}+\frac{\text { const } \cdot\|h\|^{2}}{n^{(2 \alpha-1) \sigma+1 / 2}(1+|t|)^{2(1-\alpha)}} .
$$

Положим $2 \alpha \sigma=(2 \alpha-1) \sigma+1 / 2$, тогда $\sigma=1 / 2$, следовательно, из $(2.22)$ получим, что

$$
\|u\|^{2}=\left\|R_{0}(2 n+3+i t) \theta h\right\|^{2} \leqslant n^{-\alpha}(1+|t|)^{-2(1-\alpha)} \text { const } \cdot\|h\|^{2},
$$

откуда вытекает неравенство (2.15). Аналогично из теории возмущений следует (2.16). Лемма 2.2 доказана.

Пусть $K$ - компакт в $\mathbb{R}^{2}, x \in K$, тогда, используя неравенство $(0.4)$, легко получаем, что

$$
\sum_{s=0}^{k}\left|\varphi_{s}^{(k)}(x)\right|^{2} \leqslant \text { const } \cdot \int_{0}^{1} \frac{d t}{\sqrt{t} \sqrt{1-t}} \leqslant M
$$

причем постоянная $M$ зависит только от $K$. 
Теперь докажем, что $\left|\sigma_{n}^{(1)}\right| \rightarrow 0$ при $n \rightarrow \infty$. Действительно, из $(2.14)$, пользуясь неравенствами $(2.15),(2.16)$ и $(2.23)$, имеем

$$
\begin{aligned}
\left|\sigma_{n}^{(1)}\right| & \leqslant \frac{\text { const } \cdot\|V\|_{\infty}^{3}}{n^{\alpha}} \sum_{k=0}^{\infty} \int_{-2 n-3}^{2 n+3} \frac{d t}{\left[(2(k-n)-1)^{2}+t^{2}\right](|t|+1)^{1-2 \alpha}} \\
& \leqslant \frac{\text { const } \cdot\|V\|_{\infty}^{3}}{n^{\alpha}} \sum_{k=0}^{\infty} \int_{0}^{\infty} \frac{d t}{\left[(2(k-n)-1)^{2}+t^{2}\right](t+1)^{1-2 \alpha}} .
\end{aligned}
$$

Далее, производя замену переменной $t=|2(k-n)-1| \tau$ в последнем интеграле, так как полученный интеграл $\int_{0}^{+\infty}\left(1+\tau^{2}\right)^{-1}\left(\tau+|2(n-k)-1|^{-1}\right)^{-1+2 \alpha} d \tau$ сходится, получаем, что при $0<\alpha<1 / 2$

$$
\left|\sigma_{n}^{(1)}\right| \leqslant \frac{\text { const } \cdot\|V\|_{\infty}^{3}}{n^{\alpha}} \sum_{k=0}^{\infty} \frac{1}{|2(k-n)-1|^{2-2 \alpha}} \leqslant \frac{\text { const } \cdot\|V\|_{\infty}^{3}}{n^{\alpha}}
$$

откуда следует (2.11). Для доказательства соотношения (2.12) положим в неравенстве $(2.22) \alpha=1-\varepsilon, 0<\varepsilon<1 / 4$, тогда $1 /(2(1-2 \varepsilon))<\delta<1$ и $2 \sigma(1-\varepsilon)>1 / 2+\sigma(1-2 \varepsilon)>1$, следовательно, из (2.22) вытекает, что

$$
\|u\|^{2}=\left\|R_{0}(2 n+3+i t) \theta h\right\|^{2} \leqslant \frac{\text { const } \cdot\|h\|^{2}}{(|t|+1)^{2 \varepsilon} n^{1 / 2+\sigma(1-2 \varepsilon)}} .
$$

Из теории возмущений следует, что оценка (2.24) верна и для $R(2 n+3+i t) \theta h$. Поэтому, повторяя рассуждения, примененные при оценке $\sigma_{n}^{(1)}$, для $\sigma_{n}^{(2)}$ получаем следующую оценку:

$$
\begin{aligned}
\left|\sigma_{n}^{(2)}\right| & \leqslant \frac{\text { const } \cdot(2 n+3)\|V\|_{\infty}^{3}}{n^{1 / 2}+\sigma(1-2 \varepsilon)} \sum_{k=0}^{\infty} \int_{0}^{\infty} \frac{d t}{\left[(2(k-n)-1)^{2}+t^{2}\right](t+1)^{2 \varepsilon}} \\
& \leqslant \frac{\text { const } \cdot\|V\|_{\infty}^{3}}{n^{\sigma(1-2 \varepsilon)-1 / 2}} \sum_{k=0}^{\infty} \frac{1}{|2(k-n)-1|^{1+2 \varepsilon}} \leqslant \frac{\text { const } \cdot\|V\|_{\infty}^{3}}{n^{\sigma(1-2 \varepsilon)-1 / 2}}
\end{aligned}
$$

Отсюда при $0<\varepsilon<1 / 4,1 /(2(1-2 \varepsilon))<\sigma<1$ следует (2.12).

Покажем справедливость соотношения (2.13). Пусть $z \in \Pi_{n}(\varphi)$, тогда $\left\|R_{0}(z)\right\|<(2 n+3)^{-1},\|R(z)\|<(2 n+3)^{-1}, \inf _{z \in \Pi_{n}(\varphi)}\left|\lambda_{k}-z\right|^{2}>(2(k-n)-1)^{2}+$ $(2 n+3)^{2}$. Используя эти неравенства и учитывая $(2.23)$, получаем

$$
\begin{aligned}
\left|\sigma_{n}^{(3)}\right| & =\left|-\frac{1}{2 \pi i} \int_{\Pi_{n}(\varphi)} z \sum_{k=0}^{\infty} \sum_{s=0}^{k} \frac{\left(V R_{0}(z) V R(z) V \varphi_{s}^{(k)}, \varphi_{s}^{(k)}\right)}{\left(\lambda_{k}-z\right)^{2}}\right| \\
& \leqslant \text { const } \cdot\|V\|_{\infty}^{3} \sum_{k=0}^{\infty} \frac{1}{(2(k-n)-1)^{2}+(2 n+3)^{2}} \\
& \leqslant \text { const } \cdot\|V\|_{\infty}^{3} \int_{0}^{\infty} \frac{d t}{(2(t-n)-1 / 2)^{2}+(2 n+3)^{2}} .
\end{aligned}
$$


После замены переменной $t=(n+1 / 4) \tau$ в последнем интеграле из последнего неравенства легко получим, что

$$
\left|\sigma_{n}^{(3)}\right| \leqslant \frac{\text { const } \cdot\|V\|_{\infty}^{3}}{2 n+1} \int_{0}^{\infty} \frac{d \tau}{(\tau-1)^{2}+1}
$$

Отсюда следует (2.13), так как интеграл в последнем неравенстве сходится. Теорема 2.1 доказана.

\section{§ 3. Асимптотическое представление $\mathscr{P}_{n}(x, y)$}

Введем функции $p_{k}(s, t)=f_{k}(s) f_{k}(t), f_{k}(t)$ - нормированные собственные функции одномерного гармонического осциллятора (определенные формулой $(0.3)$ ), тогда

$$
\mathscr{P}_{n}(x, y)=\sum_{l=0}^{n} p_{l}\left(x_{1}, y_{1}\right) p_{n-l}\left(x_{2}, y_{2}\right)
$$

Пусть $0<\sigma<1$, тогда ядро $\mathscr{P}_{n}(x, y)$ представим в следуюшем виде:

$$
\mathscr{P}_{n}(x, y)=\mathscr{P}^{(1)}(x, y)+\mathscr{P}^{(2)}(x, y)+\mathscr{P}^{(1)}(y, x)
$$

где

$$
\begin{aligned}
& \mathscr{P}_{n}^{(1)}(x, y)=\sum_{l=0}^{\left[n^{\sigma}\right]-1} p_{l}\left(x_{1}, y_{1}\right) p_{n-l}\left(x_{2}, y_{2}\right), \\
& \mathscr{P}_{n}^{(2)}(x, y)=\sum_{l=\left[n^{\sigma}\right]}^{n-\left[n^{\sigma}\right]} p_{l}\left(x_{1}, y_{1}\right) p_{n-l}\left(x_{2}, y_{2}\right), \\
& \mathscr{P}_{n}^{(1)}(y, x)=\sum_{l=0}^{\left[n^{\sigma}\right]-1} p_{n-l}\left(x_{1}, y_{1}\right) p_{l}\left(x_{2}, y_{2}\right) .
\end{aligned}
$$

Исследуем при $n \gg 1$ каждое слагаемое в правой части соотношения (3.1). Рассмотрим второе слагаемое $\mathscr{P}_{n}^{(2)}(x, y)$ : так как $0<\sigma<1, n \gg 1$, то $n-l \gg 1$ и $l \gg 1$, и, подставляя асимптотические формулы $(0.5)$ в $(3.3)$, после несложных преобразований получаем

$$
\mathscr{P}_{n}^{(2)}(x, y)=\widetilde{\mathscr{P}}_{n}^{(2)}(x, y)+\widehat{\mathscr{P}}_{n}^{(2)}(x, y),
$$

где

$$
\begin{aligned}
\widetilde{\mathscr{P}}_{n}^{(2)}(x, y)= & \frac{1}{\pi^{2}} \sum_{k=\left[n^{\sigma}\right]}^{n-\left[n^{\sigma}\right]}\left[g_{n}(k, a, b)+(-1)^{k} g_{n}(k, \bar{a}, b)\right. \\
& \left.+(-1)^{n-k} g_{n}(k, a, \bar{b})+(-1)^{n} g_{n}(k, \bar{a}, \bar{b})\right] \\
= & \frac{1}{\pi^{2}}\left\{F_{n}^{(1)}(a, b)+F_{n}^{(2)}(\bar{a}, b)+F_{n}^{(3)}(a, \bar{b})+(-1)^{n} F_{n}^{(1)}(\bar{a}, \bar{b})\right\}
\end{aligned}
$$


здесь и в дальнейшем $a=x_{1}-y_{1}, \bar{a}=x_{1}+y_{1}, b=x_{2}-y_{2}, \bar{b}=x_{2}+y_{2}$,

$$
\begin{gathered}
g_{n}(t, a, b)=\frac{\cos \sqrt{2 t+1} a \cos \sqrt{2(n-t)} b}{\sqrt{2 t+1} \sqrt{2(n-t)+1}} \\
\widehat{\mathscr{P}}_{n}^{(2)}(x, y)=\frac{1}{\pi^{2}} \sum_{k=\left[n^{\sigma}\right]}^{n-\left[n^{\sigma}\right]}\left\{\frac{x_{1}^{3}-y_{1}^{3}}{6} \frac{\sin \left(h_{k} a\right)\left[\cos \left(h_{n-k} b\right)+(-1)^{n-k} \cos \left(h_{n-k} \bar{b}\right)\right]}{(2 k+1) h_{n-k}}\right. \\
+\frac{x_{1}^{3}+y_{1}^{3}}{6} \frac{\sin \left(h_{k} \bar{a}\right)}{(2 k+1) h_{n-k}}\left[(-1)^{k} \cos \left(h_{n-k} b\right)+(-1)^{n} \cos \left(h_{n-k} \bar{b}\right)\right] \\
+\frac{x_{2}^{3}-y_{2}^{3}}{6} \frac{\sin \left(h_{n-k} b\right)}{h_{k}(2(n-k)+1)}\left[\cos \left(h_{k} a\right)+(-1)^{k} \cos \left(h_{n-k} \bar{a}\right)\right] \\
\left.+\frac{x_{2}^{3}+y_{2}^{3}}{6} \frac{\sin \left(h_{n-k} \bar{b}\right)}{h_{k}(2(n-k)+1)}\left[(-1)^{n-k} \cos \left(h_{k} a\right)+(-1)^{n} \cos \left(h_{k} \bar{a}\right)\right]+\widetilde{r}_{n}(k, x, y)\right\},
\end{gathered}
$$

здесь $h_{i}=\sqrt{2 i+1}$, причем $\forall(x, y) \in \operatorname{supp} V$ и $k \gg 1$

$$
\widetilde{r}_{n}(k, x, y)=O\left(\frac{1}{(2 k+1)^{\gamma}(2(n-k)+1)^{\sigma}}\right), \quad \gamma+\sigma=2, \quad \gamma=\frac{1}{2}, 1, \frac{3}{2}
$$

Рассмотрим первое слагаемое в правой части (3.6). Имеет место

ТЕОРема 3.1. Пусть $K$ - произвольный компакт в $\mathbb{R}^{2} \times \mathbb{R}^{2}$. Тогда для $n \gg 1 u(x, y) \in K$

$$
\begin{gathered}
F_{n}^{(1)}(a, b)=\frac{1}{2 \pi} J_{0}\left(\sqrt{\lambda_{n}} \sqrt{a^{2}+b^{2}}\right)-\frac{\sin \sqrt{2\left[n^{\sigma}\right]+1} a \cos \sqrt{2 n+1} b}{a \sqrt{2 n+1}} \\
-\frac{\sin \sqrt{2\left[n^{\sigma}\right]+1} b \cos \sqrt{2 n+1} a}{b \sqrt{2 n+1}}+g_{n}^{(1)}(a, b),
\end{gathered}
$$

где $J_{0}(s)$ - функция Бесселя первого рода, а $g_{n}^{(1)}(a, b)$ при каждом $\sigma \in(0,1 / 2)$ допускает оценку

$$
\left|g_{n}^{(1)}(a, b)\right| \leqslant \frac{c_{1}}{n^{1 / 2+\sigma / 2}}+\frac{c_{2}}{n^{1-3 \sigma / 2}}
$$

причем постоянные $c_{k}>0(k=1,2)$ зависят только от $K u \sigma$.

ДокАЗАТЕльство. Так как

$$
F_{n}^{(1)}(a, b)=\sum_{k=\left[n^{\sigma}\right]}^{n-\left[n^{\sigma}\right]} g_{n}(k, a, b)
$$


применяя интегральное приближение суммы (3.10), а именно формулу (0.9), получаем, что

$$
\begin{aligned}
F_{n}^{(1)}(a, b)= & \int_{\left[n^{\sigma}\right]}^{n-\left[n^{\sigma}\right]+1} g_{n}(t, a, b) d t-\frac{1}{2}\left[g_{n}\left(n-\left[n^{\sigma}\right]+1, a, b\right)-g_{n}\left(\left[n^{\sigma}\right], a, b\right)\right] \\
& -\frac{1}{2} \sum_{k=\left[n^{\sigma}\right]}^{n-\left[n^{\sigma}\right]} \int_{k}^{k+1}\left[(t-k-1)^{2}+(t-k-1)\right] g_{n}^{\prime \prime}(t, a, b) d t .
\end{aligned}
$$

Далее, из (3.7) легко следует, что при $n \gg 1$

$$
\left|g_{n}\left(n-\left[n^{\sigma}\right]+1, a, b\right)\right| \leqslant \frac{\text { const }}{n^{1 / 2+\sigma / 2}}, \quad\left|g_{n}\left(\left[n^{\sigma}\right], a, b\right)\right| \leqslant \frac{\text { const }}{n^{1 / 2+\sigma / 2}}
$$

Для оценки последнего слагаемого в (3.11) заметим, что

$$
\begin{aligned}
\max _{k \leqslant t \leqslant k+1}\left|g_{n}^{\prime \prime}(t, a, b)\right|= & O\left(\frac{1}{(2 k+1)^{3 / 2} \sqrt{2(n-k)+1}}\right) \\
& +O\left(\frac{1}{\sqrt{2 k+1}(2(n-k)+1)^{3 / 2}}\right) \\
& +O\left(\frac{1}{(2 k+1)(2(n-k)+1)}\right),
\end{aligned}
$$

следовательно,

$$
\left|\sum_{k=\left[n^{\sigma}\right]}^{n-\left[n^{\sigma}\right]} \int_{k}^{k+1}\left[(t-k-1)^{2}+(t-k-1)\right] g_{n}^{\prime \prime}(t, a, b) d t\right| \leqslant \frac{\text { const }}{n^{1 / 2+\sigma / 2}}
$$

Известно [9; гл. $6, \S 2$, формула $(2.41) ; \S 4$, формула (4.28)], что при $\lambda>0$

$$
\int_{0}^{\lambda} \frac{\cos \sqrt{s} a \cos \sqrt{\lambda-s} b}{\sqrt{s} \sqrt{\lambda-s}} d s=\pi J_{0}\left(\sqrt{\lambda} \sqrt{a^{2}+b^{2}}\right)
$$

следовательно, из (3.11)-(3.13) вытекает

$$
\begin{aligned}
F_{n}^{(1)}(a, b)= & \frac{\pi}{2} J_{0}\left(\sqrt{\lambda} \sqrt{a^{2}+b^{2}}\right) \\
& -\left(\int_{-1 / 2}^{\left[n^{\sigma}\right]}+\int_{n-\left[n^{\sigma}\right]+1}^{n+1 / 2}\right) g_{n}(t, a, b) d t+O\left(\frac{1}{n^{1 / 2+\sigma / 2}}\right) .
\end{aligned}
$$

Из этой формулы следует теорема 3.1 , если покажем, что справедлива 
ЛЕмма 3.1. Пусть $K$ - произвольный компакт в $\mathbb{R}^{2} \times \mathbb{R}^{2}$. Тогда при $0<\sigma<1 / 2, n \gg 1 u(x, y) \in K$

$$
\begin{aligned}
\int_{-1 / 2}^{\left[n^{\sigma}\right]} g_{n}(t, a, b) d t & =\frac{\sin \sqrt{2\left[n^{\sigma}\right]+1} a \cos \sqrt{2 n+1} b}{a \sqrt{2 n+1}}+R_{n}^{(1)}(a, b), \\
\int_{n-\left[n^{\sigma}\right]+1}^{n+1 / 2} g_{n}(t, a, b) d t & =\frac{\sin \sqrt{2\left[n^{\sigma}\right]+1} b \cos \sqrt{2 n+1} a}{b \sqrt{2 n+1}}+R_{n}^{(2)}(a, b),
\end{aligned}
$$

причем

$$
\left|R_{n}^{(1)}(a, b)\right| \leqslant \frac{c_{1}}{n^{1-3 \sigma / 2}}, \quad\left|R_{n}^{(2)}(a, b)\right| \leqslant \frac{c_{2}}{n^{1-3 \sigma / 2}},
$$

$c_{k}>0$ и зависят только от $\sigma$ и K.

ДокАЗАТЕЛЬСтво. Докажем (3.15), а (3.16) доказывается аналогично. Так как $-1 / 2 \leqslant t \leqslant\left[n^{\sigma}\right]$ и $n \gg 1$, то

$$
\begin{gathered}
\cos \sqrt{2(n-t)+1} b=\cos \sqrt{2 n+1} b+r_{n}^{(1)}(t, b) \\
\frac{1}{\sqrt{2(n-t)+1}}=\frac{1}{\sqrt{2 n+1}}+r_{n}^{(2)}(t)
\end{gathered}
$$

где

$$
\begin{aligned}
r_{n}^{(1)}(t, b)= & 2 \sin \frac{t b}{\sqrt{2 n+1}+\sqrt{2(n-t)+1}} \\
& \times \sin \left(2 n+1-\frac{t b}{\sqrt{2 n+1}+\sqrt{2(n-t)+1}}\right) \\
r_{n}^{(2)}(t)= & \frac{2 t}{\sqrt{2 n+1} \sqrt{2(n-t)+1}(\sqrt{2 n+1}+\sqrt{2(n-t)+1})}
\end{aligned}
$$

Легко убедиться, что для $(x, y) \in K$ и $\sigma \in(0,1 / 2)$

$$
\left|r_{n}^{(1)}(t, b)\right| \leqslant \frac{\text { const }}{n^{1 / 2-\sigma}}, \quad\left|r_{n}^{(2)}(t)\right| \leqslant \frac{\text { const }}{n^{3 / 2-\sigma}} .
$$

Подставив (3.18) в (3.7), так как

$$
\frac{1}{2} \int_{0}^{2\left[n^{\sigma}\right]+1} \frac{\cos \sqrt{s} a}{\sqrt{s}} d s=\frac{\sin \sqrt{2\left[n^{\sigma}\right]+1} a}{a}
$$

получим (3.15), причем функция $R_{n}^{(1)}(a, b)$ задается следующей формулой:

$$
\begin{aligned}
R_{n}^{(1)}(a, b)= & \int_{-1 / 2}^{\left[n^{\sigma}\right]} \frac{\cos \sqrt{2 t+1} a}{\sqrt{2 t+1}} \\
& \times\left\{\cos \sqrt{2 n+1} b r_{n}^{(2)}(t)+\frac{r_{n}^{(1)}(t, b)}{\sqrt{2 n+1}}+r_{n}^{(1)}(t, b) r_{n}^{(2)}(t)\right\} d t .
\end{aligned}
$$


Отсюда в силу неравенств (3.19) следует первое из неравенств (3.17). Лемма 3.1 доказана.

Таким образом, лемма 3.1 совместно с формулой (3.14) завершают доказательство теоремы 3.1.

Далее, для четвертого слагаемого в правой части (3.6) асимптотическое представление получается из (3.9) умножением на $(-1)^{n}$ и заменой $a, b$ на $\bar{a}, \bar{b}$ соответственно. Теперь исследуем функции $F_{n}^{(2)}(\bar{a}, b)$ и $F_{n}^{(3)}(a, \bar{b})$ в $(3.6)$. Так как

$$
F_{n}^{(2)}(\bar{a}, b)=\sum_{k=\left[n^{\sigma}\right]}^{n-\left[n^{\sigma}\right]}(-1)^{k} g_{n}(k, \bar{a}, b), \quad F_{n}^{(3)}(a, \bar{b})=\sum_{k=\left[n^{\sigma}\right]}^{n-\left[n^{\sigma}\right]}(-1)^{n-k} g_{n}(k, a, \bar{b}),
$$

разбиваем правые части на суммы четных и нечетных слагаемых. Применяя рассуждения и выкладки теоремы 3.1 к каждой полученной сумме, убеждаемся, что главные части в асимптотическомпредставлении типа (3.9) для четных и нечетных слагаемых взаимно уничтожаются. И справедливы оценки:

$$
\left|F_{n}^{(2)}(\bar{a}, b)\right| \leqslant \frac{\text { const }}{n^{1 / 2+\sigma / 2}}, \quad\left|F_{n}^{(3)}(a, \bar{b})\right| \leqslant \frac{\text { const }}{n^{1 / 2+\sigma / 2}} .
$$

Для получения асимптотического представления $\mathscr{P}_{n}^{(2)}(x, y)$ оценим $\widehat{\mathscr{P}}_{n}^{(2)}(x, y)$. Справедлива

Лемма 3.2. Пусть $K-$ компакт в $\mathbb{R}^{2} \times \mathbb{R}^{2},(x, y) \in K$. Тогда для $n \gg 1$ при каждом $\sigma \in(0,1)$ справедлива следующая оченка

$$
\left|\widehat{\mathscr{P}}_{n}^{(2)}(x, y)\right| \leqslant \frac{\text { const }}{n^{1 / 2+\sigma / 2}} .
$$

ДокАЗАТЕльство. Покажем справедливость оценки (3.21) для первого слагаемого в (3.8), остальные слагаемые рассматриваются аналогично. Итак, имеем

$$
\begin{gathered}
G_{n}(a, b)=\sum_{k=\left[n^{\sigma}\right]}^{n-\left[n^{\sigma}\right]} q_{n}(k, a, b), \\
q_{n}(k, a, b)=\frac{x_{1}^{3}-y_{1}^{3}}{\pi^{2}} \frac{\sin \sqrt{2 k+1} a \cos \sqrt{2(n-k)+1} b}{(2 k+1) \sqrt{2(n-k)+1}} .
\end{gathered}
$$

Применив лемму 0.1 к сумме (3.22), получим

$$
G_{n}(a, b)=\int_{k=\left[n^{\sigma}\right]}^{n-\left[n^{\sigma}\right]+1} q_{n}(t, a, b) d t+\sum_{k=\left[n^{\sigma}\right]}^{n-\left[n^{\sigma}\right]} \int_{k}^{k+1}(t-k-1) q_{n}^{\prime}(t, a, b) d t .
$$

Оценим интеграл в (3.24). Для этого интегрируем по частям, что возможно за счет множителя $x_{1}^{3}-y_{1}^{3}$ в (3.23). Тогда легко показать, что

$$
\int_{k=\left[n^{\sigma}\right]}^{n-\left[n^{\sigma}\right]+1} q_{n}(t, a, b) d t=O\left(\frac{1}{n^{1 / 2+3 \sigma / 2}}\right) .
$$


Далее, вычислив $q_{n}^{\prime}(t, a, b)$, после несложных преобразований получим

$$
\left|\sum_{k=\left[n^{\sigma}\right]}^{n-\left[n^{\sigma}\right]} \int_{k}^{k+1}(t-k-1) q_{n}^{\prime}(t, a, b) d t\right| \leqslant \frac{\text { const }}{n^{1 / 2+\sigma / 2}} .
$$

Отсюда в силу (3.24) и (3.23) вытекает оценка (3.21) для первого слагаемого в (3.8). Лемма доказана.

Таким образом, из теоремы 3.1 , оценок (3.20) и леммы 3.2 в силу (3.5) и (3.6) окончательно имеем

$$
\begin{aligned}
\mathscr{P}_{n}^{(2)}(x, y)= & \frac{1}{2 \pi} J_{0}\left(\sqrt{\lambda_{n}}|x-y|\right)+\frac{(-1)^{n}}{2 \pi} J_{0}\left(\sqrt{\lambda_{n}}|x+y|\right) \\
& -\frac{\cos \left(h_{n} b\right) \sin \left(l_{n} a\right)}{\pi^{2} a h_{n}}-\frac{\sin \left(l_{n} b\right) \cos \left(h_{n} a\right)}{\pi^{2} b h_{n}} \\
& +\frac{(-1)^{n}}{\pi^{2}}\left\{\frac{\cos \left(h_{n} \bar{b}\right) \sin \left(l_{n} \bar{a}\right)}{\bar{a} h_{n}}+\frac{\cos \left(h_{n} \bar{a}\right) \sin \left(l_{n} \bar{b}\right)}{\bar{b} h_{n}}\right\}+Q_{n}(x, y),
\end{aligned}
$$

здесь и в дальнейшем $h_{n}=\sqrt{2 n+1}, l_{n}=\sqrt{2\left[n^{\sigma}\right]+1}$, причем

$$
\left|Q_{n}(x, y)\right| \leqslant \frac{c_{1}}{n^{1 / 2+\sigma / 2}}+\frac{c_{2}}{n^{1-3 \sigma / 2}}
$$

$c_{k}>0$ и зависят только от $\sigma$ и $K$.

Теперь исследуем асимптотику функций $\mathscr{P}_{n}^{(1)}(x, y)$ и $\mathscr{P}_{n}^{(1)}(y, x)$, определенных формулами (3.2) и (3.4). А именно справедлива

Лемма 3.3. Пусть $K-$ компакт в $\mathbb{R}^{2} \times \mathbb{R}^{2},(x, y) \in K$. Тогда для $n \gg 1$

$$
\begin{aligned}
& \mathscr{P}_{n}^{(1)}(x, y)=\frac{1}{\pi^{2}}\left\{\frac{\cos \left(h_{n} b\right) \sin \left(l_{n} a\right)}{a h_{n}}+\frac{(-1)^{n} \sin \left(l_{n} \bar{a}\right) \cos \left(h_{n} \bar{b}\right)}{\bar{a} h_{n}}\right\}+\Gamma_{n}(x, y) \\
& \mathscr{P}_{n}^{(1)}(y, x)=\frac{1}{\pi^{2}}\left\{\frac{\cos \left(h_{n} a\right) \sin \left(l_{n} b\right)}{b h_{n}}+\frac{(-1)^{n} \sin \left(l_{n} \bar{b}\right) \cos \left(h_{n} \bar{a}\right)}{\bar{b} h_{n}}\right\}+\Gamma_{n}(x, y)
\end{aligned}
$$

Причем при каждом $\sigma \in(0,1 / 2)$

$$
\left|\Gamma_{n}(x, y)\right| \leqslant \frac{c_{1}}{n^{1 / 2+\sigma / 2}}+\frac{c_{2}}{n^{1-3 \sigma / 2}}
$$

$c_{k}>0(k=1,2)$ и зависят только от $\sigma$ и $K$. 
ДокАЗАТЕЛЬСтво. Установим справедливость формулы (3.28). Из представления (3.2) следует, что $n-l \gg 1$ при $n \gg 1$, поэтому, используя $(0.5)$, получаем

$$
\begin{aligned}
p_{n-l}\left(x_{2}, y_{2}\right)= & \frac{1}{\pi \sqrt{2(n-l)+1}} \cos \left(\sqrt{2(n-l)+1}\left(x_{2}-y_{2}\right)\right) \\
& +\frac{1}{\pi \sqrt{2(n-l)+1}}(-1)^{n} \cos \left(\sqrt{2(n-l)+1}\left(x_{2}+y_{2}\right)\right) \\
& +\widehat{p}_{n-l}\left(x_{2}, y_{2}\right),
\end{aligned}
$$

причем

$$
\left|\widehat{p}_{n-l}\left(x_{2}, y_{2}\right)\right|<\text { const } \cdot(2(n-l)+1)^{-1} .
$$

Отсюда, применяя преобразования (3.18), получаем, что

$$
p_{n-l}\left(x_{2}, y_{2}\right)=\frac{b \cos \sqrt{2 n+1}}{\pi \sqrt{2 n+1}}+\frac{(-1)^{n-l} \cos \bar{b} \sqrt{2 n+1}}{\pi \sqrt{2 n+1}}+r_{n}(l, b, \bar{b})
$$

причем

$$
\left|r_{n}(l, b, \bar{b})\right| \leqslant \frac{\text { const } \cdot l}{n} .
$$

Подставляя (3.31) в (3.2), имеем

$$
\begin{aligned}
\mathscr{P}_{n}^{(1)}(x, y)= & \frac{\cos b \sqrt{2 n+1}}{\pi \sqrt{2 n+1}} \sum_{l=0}^{\left[n^{\sigma}\right]-1} p_{l}\left(x_{1}, y_{1}\right) \\
& +\frac{(-1)^{n} \cos \bar{b} \sqrt{2 n+1}}{\pi \sqrt{2 n+1}} \sum_{l=0}^{\left[n^{\sigma}\right]-1}(-1)^{l} p_{l}\left(x_{1}, y_{1}\right) \\
& +\sum_{l=0}^{\left[n^{\sigma}\right]-1} p_{l}\left(x_{1}, y_{1}\right) r_{n}(l, b, \bar{b}) .
\end{aligned}
$$

Исследуем каждое слагаемое в (3.33). Из формулы Дарбу-Кристоффеля [7; гл. 3, п. 3.2, формула (3.2.3)] следует, что

$$
\sum_{l=0}^{\left[n^{\sigma}\right]-1} p_{l}\left(x_{1}, y_{1}\right)=\frac{\sqrt{\left[n^{\sigma}\right]}}{\sqrt{2}} \frac{f_{\left[n^{\sigma}\right]}\left(x_{1}\right) f_{\left[n^{\sigma}\right]-1}\left(y_{1}\right)-f_{\left[n^{\sigma}\right]}\left(y_{1}\right) f_{\left[n^{\sigma}\right]-1}\left(x_{1}\right)}{x_{1}-y_{1}} .
$$

Отсюда, так как $\left[n^{\sigma}\right] \gg 1$, используя $(0.5)$, после несложных преобразований получаем

$$
\sum_{l=0}^{\left[n^{\sigma}\right]-1} p_{l}\left(x_{1}, y_{1}\right)=\frac{1}{\pi} \frac{\sin \left(\sqrt{2\left[n^{\sigma}\right]+1}\left(x_{1}-y_{1}\right)\right)}{x_{1}-y_{1}}+r_{n}\left(x_{1}, y_{1}\right) .
$$

Отметим, что

$$
\left|r_{n}\left(x_{1}, y_{1}\right)\right| \leqslant \frac{\text { const }}{n^{\sigma / 2}}
$$


Далее, так как $f_{l}(-x)=(-1)^{l} f_{l}(x)$, из (3.35) имеем

$$
\begin{aligned}
\sum_{l=0}^{\left[n^{\sigma}\right]-1}(-1)^{l} p_{l}\left(x_{1}, y_{1}\right) & =\sum_{l=0}^{\left[n^{\sigma}\right]-1} p_{l}\left(x_{1},-y_{1}\right) \\
& =\frac{1}{\pi} \frac{\sin \left(\sqrt{2\left[n^{\sigma}\right]+1}\left(x_{1}+y_{1}\right)\right)}{x_{1}+y_{1}}+r_{n}\left(x_{1},-y_{1}\right) .
\end{aligned}
$$

А для третьего слагаемого в (3.33) из глобальной оценки (0.4) и (3.32) вытекает оценка

$$
\left|\sum_{l=0}^{\left[n^{\sigma}-1\right]} p_{l}\left(x_{1}, y_{1}\right) r_{n}(l, b, \bar{b})\right| \leqslant \frac{\text { const }}{n^{1-3 \sigma / 2}} .
$$

Итак, из (3.33), (3.35) и (3.37) вытекает (3.28), а неравенство (3.30) следует из (3.36) и (3.38). Формула (3.29) доказьвается аналогично. Лемма 3.3 доказана.

Теперь из (3.1), (3.26), (3.27) и леммы 3.3 заключаем, что имеет место основное утверждение этого параграфа:

ТеОРема 3.2. Пусть $K-$ компакт в $\mathbb{R}^{2} \times \mathbb{R}^{2}$. Тогда для $n \gg 1 u(x, y) \in K$

$$
\mathscr{P}_{n}(x, y)=\frac{J_{0}\left(\sqrt{\lambda_{n}}|x-y|\right)}{2 \pi}+\frac{(-1)^{n} J_{0}\left(\sqrt{\lambda_{n}}|x+y|\right)}{2 \pi}+r_{n}(x, y),
$$

где $J_{0}(s)$ - функиия Бесселя 1-го рода, а $r_{n}(x, y)$ при каждом $\sigma \in(0,1 / 2)$ допускает оченку

$$
\left|r_{n}(x, y)\right| \leqslant \frac{c_{1}}{n^{1 / 2+\sigma / 2}}+\frac{c_{2}}{n^{1-3 \sigma / 2}},
$$

причем $c_{k}>0(k=1,2)$ и зависят только от $\sigma$ и K.

\section{§4. Асимптотика ядра $B_{n}(x, y)$}

Из определения оператора $B_{n}$ (см. (2.3)) следует, что

$$
\mathscr{B}_{n}(x, y)=\sum_{m \neq n}\left(\lambda_{m}-\lambda_{n}\right)^{-1} \mathscr{P}_{m}(x, y) .
$$

Пусть $0<\sigma<1$, тогда при $n \gg 1$, используя (3.39), представим функцию $\mathscr{B}_{n}(x, y)$ в следующем виде

$$
\begin{aligned}
\mathscr{B}_{n}(x, y)= & \sum_{m=0}^{\left[n^{\sigma}\right]-1} \frac{\mathscr{P}_{m}(x, y)}{\lambda_{m}-\lambda_{n}}+\sum_{\substack{m=\left[n^{\sigma}\right] \\
m \neq n}}^{\infty} \frac{1}{\lambda_{m}-\lambda_{n}} \\
& \times\left[\frac{J_{0}\left(\sqrt{\lambda_{m}}|x-y|\right)}{2 \pi}+\frac{(-1)^{m} J_{0}\left(\sqrt{\lambda_{m}}|x+y|\right)}{2 \pi}+r_{m}(x, y)\right] .
\end{aligned}
$$

Исследуем каждое слагаемое в (4.1). Имеет место 
Лемма 4.1. Пусть $K$ - произвольный компакт в $\mathbb{R}^{2} \times \mathbb{R}^{2}$ и $0<\varepsilon<1 / 2$. Тогда при $n \gg 1 u(x, y) \in K$

$$
\begin{gathered}
\sum_{m=0}^{\left[n^{\sigma}\right]-1}\left(\lambda_{m}-\lambda_{n}\right)^{-1} \mathscr{P}_{m}(x, y)=O\left(n^{-1+\sigma}\right), \quad 0<\sigma<1 \\
\sum_{\substack{m=\left[n^{\sigma}\right] \\
m \neq n}}^{\infty}\left(\lambda_{m}-\lambda_{n}\right)^{-1} r_{m}(x, y)=O\left(\frac{\ln n}{n^{1 / 2+\varepsilon / 2}}\right)+O\left(\frac{\ln n}{n^{1-(3 / 2) \varepsilon}}\right) .
\end{gathered}
$$

ДокАЗАТЕльство. Для доказательства оценки (4.2) заметим, что $\lambda_{m}-\lambda_{n}=$ $(2 n)^{-1}-m(2 n(m-n))^{-1}$. Поэтому имеем

$$
\sum_{m=0}^{\left[n^{\sigma}\right]-1} \frac{\mathscr{P}_{m}(x, y)}{\lambda_{m}-\lambda_{n}}=\frac{1}{2 n} \sum_{m=0}^{\left[n^{\sigma}\right]-1} \mathscr{P}_{m}(x, y)+\frac{1}{2 n} \sum_{m=0}^{\left[n^{\sigma}\right]-1} \frac{m \mathscr{P}_{m}(x, y)}{n-m}
$$

В силу глобальной оценки $(0.4)$ имеем $\left|\mathscr{P}_{m}(x, y)\right| \leqslant$ const. Отсюда и из последнего равенства следует (4.2).

Соотношение (4.3) легко вытекает из (3.40), если в левой части (4.3) по отдельности оценить суммы от $\left[n^{\sigma}\right]$ до $n-1$ и от $n+1$ до бесконечности. Лемма 4.1 доказана.

Далее, оставшиеся два слагаемых в (4.1) обозначим через

$$
\begin{aligned}
& \mathscr{B}_{n}^{(1)}(x, y)=\frac{1}{2 \pi} \sum_{\substack{m=\left[n^{\sigma}\right] \\
m \neq n}}^{\infty} \frac{J_{0}\left(\sqrt{\lambda_{m}}|x-y|\right)}{\lambda_{m}-\lambda_{n}}, \\
& \mathscr{B}_{n}^{(2)}(x, y)=\frac{1}{2 \pi} \sum_{\substack{m=\left[n^{\sigma}\right] \\
m \neq n}}^{\infty} \frac{(-1)^{m} J_{0}\left(\sqrt{\lambda_{m}}|x+y|\right)}{\lambda_{m}-\lambda_{n}} .
\end{aligned}
$$

Изучим асимптотическое поведение функции $\mathscr{B}_{n}^{(1)}(x, y)$, для этого представим ее в виде суммы следующих функций

$$
\begin{aligned}
& \mathscr{B}_{n}^{(1,1)}(x, y)=\frac{1}{2 \pi} \sum_{m=\left[n^{\sigma}\right]}^{n-\left[n^{\sigma}\right]-1} \frac{J_{0}\left(\sqrt{\lambda_{m}}|x-y|\right)}{\lambda_{m}-\lambda_{n}}+\frac{1}{2 \pi} \sum_{m=n+\left[n^{\sigma}\right]+1}^{\infty} \frac{J_{0}\left(\sqrt{\lambda_{m}}|x-y|\right)}{\lambda_{m}-\lambda_{n}}, \\
& \mathscr{B}_{n}^{(1,2)}(x, y)=\frac{1}{2 \pi} \sum_{\substack{m=n-\left[n^{\sigma}\right] \\
m \neq n}}^{n+\left[n^{\sigma}\right]} \frac{J_{0}\left(\sqrt{\lambda_{m}}|x-y|\right)}{\lambda_{m}-\lambda_{n}} .
\end{aligned}
$$


Teорема 4.1. Пусть $K-$ компакт в $\mathbb{R}^{2} \times \mathbb{R}^{2} u 0<\sigma<1 / 2$. Тогда при $n \gg 1 u(x, y) \in K$

$$
\begin{aligned}
\mathscr{B}_{n}^{(1,1)}(x, y)=- & \frac{1}{4} Y_{0}\left(\sqrt{\lambda_{n}}|c|\right)-\frac{|c|}{4 \pi} \frac{J_{0}^{\prime}\left(\sqrt{\lambda_{n}}|c|\right)}{\sqrt{\lambda_{n}}}\left(2\left[n^{\sigma}\right]+1\right)+\frac{|c|}{8 \pi} \frac{J_{0}^{\prime}\left(\sqrt{\lambda_{n}}|c|\right)}{\sqrt{\lambda_{n}}} \\
& +O\left(\frac{1}{n^{7 / 4-2 \sigma}}\right)+O\left(\frac{1}{n^{1-\sigma}}\right)+O\left(\frac{1}{n^{3 / 4+\sigma}}\right)+O\left(\frac{1}{n^{3 \sigma}}\right)
\end{aligned}
$$

где $c=x-y, J_{0}(s), Y_{0}(s)$ - функиии Бесселя первого и второго рода соответственно.

ДоКАЗАТЕЛЬСТвО. Положим $|c|=|x-y|$ и, используя равенства

$$
\begin{gathered}
\int_{0}^{\infty} \frac{J_{0}(\sqrt{t}|c|) d t}{t-z}=\pi i H_{0}^{(1)}(\sqrt{z}|c|), \quad z \notin[0,+\infty) \\
\pi i H_{0}^{(1)}(\sqrt{z}|c|)=\pi i J_{0}(\sqrt{z}|c|)-\pi Y_{0}(\sqrt{z}|c|)
\end{gathered}
$$

[9; гл. $6, \S 2$, п. 8, формула (2.34)], [10; гл. 3 , п. 3.7, формула (8)], преобразуем следующий интеграл:

$$
\begin{aligned}
& I_{n}(|c|)=\left(\int_{\lambda_{\left[n^{\sigma}\right]-1}}^{\lambda_{n-\left[n^{\sigma}\right]-1}}+\int_{\lambda_{n+\left[n^{\sigma}\right]+1}}^{\infty}\right) \frac{J_{0}(\sqrt{t}|c|)}{t-\lambda_{n}} d t \\
& =\lim _{\varepsilon \rightarrow 0}\left[\int_{0}^{\infty} \frac{J_{0}(\sqrt{t}|c|)}{t-\lambda_{n}-i \varepsilon} d t-\int_{0}^{\lambda_{\left[n^{\sigma}\right]-1}} \frac{J_{0}(\sqrt{t}|c|)}{t-\lambda_{n}-i \varepsilon} d t\right. \\
& \left.-\int_{\lambda_{n-\left[n^{\sigma}\right]-1}}^{\lambda_{n+\left[n^{\sigma}\right]+1}} \frac{J_{0}(\sqrt{t}|c|)}{t-\lambda_{n}-i \varepsilon} d t\right] .
\end{aligned}
$$

Воспользуемся равенствами (4.9) и (4.10) при $z=\lambda_{n}+i \varepsilon$ и, преобразуя третий интеграл в правой части (4.11) с помощью равенства

$$
\lim _{\varepsilon \rightarrow 0} \int_{\lambda_{n-\left[n^{\sigma}\right]-1}}^{\lambda_{n+\left[n^{\sigma}\right]+1}} \frac{J_{0}\left(\sqrt{\lambda_{n}}|c|\right)}{t-\lambda_{n}-i \varepsilon} d t=\pi i J_{0}\left(\sqrt{\lambda_{n}}|c|\right)
$$

получаем

$$
\begin{aligned}
I_{n}(|c|)= & -\pi Y_{0}\left(\lambda_{n}|c|\right)-\int_{0}^{\lambda_{\left[n^{\sigma}\right]-1}} \frac{J_{0}(\sqrt{t}|c|)}{t-\lambda_{n}} d t \\
& -\int_{\lambda_{n-\left[n^{\sigma}\right]-1}}^{\lambda_{n+\left[n^{\sigma}\right]+1}} \frac{J_{0}(\sqrt{t}|c|)-J_{0}\left(\sqrt{\lambda_{n}}|c|\right)}{t-\lambda_{n}} d t .
\end{aligned}
$$

Далее, можно применить лемму 0.1 к суммам в правой части (4.6), так как функция $\gamma(t)=\frac{J_{0}(\sqrt{t}|c|)}{t-\lambda_{n}}$ удовлетворяет условиям леммы 0.1 в указанных пределах суммирования. Отметим, что для первого слагаемого (4.6) в интегральном приближении пределы интегрирования берем от $\lambda_{m-1}$ до $\lambda_{m}$, а для второго - от $\lambda_{m}$ до $\lambda_{m+1}$, 
поэтому первое интегральное слагаемое в формуле $(0.6)$ совпадает с $I_{n}(|c|)$. Следовательно, из леммы 0.1 и (4.12) для функции $\mathscr{B}_{n}^{(1,1)}(x, y)$ получаем следующее представление:

$$
\begin{aligned}
\mathscr{B}_{n}^{(1,1)}(x, y)= & -\frac{1}{4} Y_{0}\left(\sqrt{\lambda_{n}}|c|\right)-\frac{1}{4 \pi} \int_{0}^{\lambda_{\left[n^{\sigma}\right]-1}} \frac{J_{0}(\sqrt{t}|c|)}{t-\lambda_{n}} d t \\
& -\frac{1}{4 \pi} \int_{\lambda_{n-\left[n^{\sigma}\right]-1}}^{\lambda_{n+\left[n^{\sigma}\right]+1}} \frac{J_{0}(\sqrt{t}|c|)-J_{0}\left(\sqrt{\lambda_{n}}|c|\right)}{t-\lambda_{n}} d t \\
& +\frac{1}{2}\left\{\gamma_{n}\left(\lambda_{n-\left[n^{\sigma}\right]-1}\right)+\gamma_{n}\left(\lambda_{n+\left[n^{\sigma}\right]+1}\right)-\gamma_{n}\left(\lambda_{\left[n^{\sigma}\right]-1}\right)-\gamma_{n}(\infty)\right\} \\
& +\frac{1}{6}\left\{\gamma_{n}^{\prime}\left(\lambda_{n-\left[n^{\sigma}\right]-1}\right)-\gamma_{n}^{\prime}\left(\lambda_{n+\left[n^{\sigma}\right]+1}\right)-\gamma_{n}^{\prime}\left(\lambda_{\left[n^{\sigma}\right]-1}\right)+\gamma_{n}^{\prime}(\infty)\right\} \\
& +\Gamma_{n}\left(\left[n^{\sigma}\right], n-\left[n^{\sigma}\right]-1\right)+\Gamma_{n}\left(n+\left[n^{\sigma}\right]+1, \infty\right),
\end{aligned}
$$

где $\gamma_{n}(t)=\frac{J_{0}(\sqrt{t}|c|)}{t-\lambda_{n}}$, а $\Gamma_{n}(a, b)$ определяется формулой $(0.7)$.

Изучим каждое слагаемое в правой части (4.13). Так как $\left|J_{0}(\sqrt{t}|c|)\right| \leqslant$ const, нетрудно заметить, что при $n \gg 1$

$$
\int_{0}^{\lambda_{\left[n^{\sigma}\right]-1}} \frac{J_{0}(\sqrt{t}|c|)}{t-\lambda_{n}}=O\left(\frac{1}{n^{1-\sigma}}\right) .
$$

Для второго интеграла в (4.13) справедливо следуюшее утверждение.

ЛЕмма 4.2. В условиях теоремы 4.1

$$
\begin{aligned}
& \int_{\lambda_{n-\left[n^{\sigma}\right]-1}}^{\lambda_{n+\left[n^{\sigma}\right]+1}} \frac{J_{0}(\sqrt{t}|c|)-J_{0}\left(\sqrt{\lambda_{n}}|c|\right)}{t-\lambda_{n}} d t \\
& \quad=\frac{|c| J_{0}^{\prime}\left(\sqrt{\lambda_{n}}|c|\right)}{\sqrt{\lambda_{n}}} 2\left(\left[n^{\sigma}\right]+1\right)+O\left(\frac{1}{n^{7 / 4-3 \sigma}}\right) .
\end{aligned}
$$

ДокАЗАТЕЛЬСТво. Производя замену переменной $t-\lambda_{n}=s$ в левой части (4.15), получаем

$$
\begin{aligned}
& \int_{\lambda_{n-\left[n^{\sigma}\right]-1}}^{\lambda_{n+\left[n^{\sigma}\right]+1}} \frac{J_{0}(\sqrt{t}|c|)-J_{0}\left(\sqrt{\lambda_{n}}|c|\right)}{t-\lambda_{n}} d t \\
& =\int_{0}^{2\left(\left[n^{\sigma}\right]+1\right)} \frac{J_{0}\left(\sqrt{\lambda_{n}+s}|c|\right)-J_{0}\left(\sqrt{\lambda_{n}-s}|c|\right)}{s} d s .
\end{aligned}
$$

Далее, обозначив

$$
\psi_{n}(s)=J_{0}\left(\sqrt{\lambda_{n}+s}|c|\right)-J_{0}\left(\sqrt{\lambda_{n}-s}|c|\right)
$$

и применив формулу Тейлора $\left(s \ll \lambda_{n}\right)$, а также неравенство [7; гл. 7, теоремa 7.31.2]

$$
x^{1 / 2}\left|J_{\nu}(x)\right| \leqslant \text { const }, \quad x \geqslant 0, \quad \nu \geqslant-1 / 2,
$$


получим, что

$$
\psi_{n}(s)=\frac{|c| J_{0}^{\prime}\left(\sqrt{\lambda_{n}}|c|\right)}{\sqrt{\lambda_{n}}} s+O\left(\frac{s^{3}}{\lambda_{n}^{7 / 4}}\right) .
$$

Следовательно, доказьваемое утверждение непосредственно вытекает из (4.19) и (4.16). Лемма 4.2 доказана.

Из определения функции $\gamma_{n}(t)$ легко следует, что при $n \gg 1$

$$
\gamma_{n}\left(\lambda_{\left[n^{\sigma}\right]-1}\right)=O\left(\frac{1}{n}\right), \quad \gamma_{n}(\infty)=\lim _{t \rightarrow \infty} \gamma_{n}(t)=0
$$

Заметим, что

$$
\gamma_{n}\left(\lambda_{n-\left[n^{\sigma}\right]-1}\right)+\gamma_{n}\left(\lambda_{n+\left[n^{\sigma}\right]+1}\right)=\frac{\psi_{n}\left(2\left(\left[n^{\sigma}\right]+1\right)\right)}{2\left(\left[n^{\sigma}\right]+1\right)},
$$

откуда в силу (4.19) получим

$$
\gamma_{n}\left(\lambda_{n-\left[n^{\sigma}\right]-1}\right)+\gamma_{n}\left(\lambda_{n+\left[n^{\sigma}\right]+1}\right)=\frac{|c| J_{0}^{\prime}\left(\sqrt{\lambda_{n}}|c|\right)}{\sqrt{\lambda_{n}}}+O\left(\frac{1}{n^{7 / 4-2 \sigma}}\right) .
$$

С целью дальнейшего исследования правой части (4.13) докажем следующую лемму.

Лемма 4.3. Пусть $0<\sigma<1$. Тогда при $n \gg 1 u(x, y) \in K$

$$
\begin{gathered}
\gamma_{n}^{\prime}\left(\lambda_{n-\left[n^{\sigma}\right]-1}\right)-\gamma_{n}^{\prime}\left(\lambda_{n+\left[n^{\sigma}\right]+1}\right)=\left(\frac{1}{n^{3 / 4+\sigma}}\right) \\
\gamma_{n}^{\prime}\left(\lambda_{\left[n^{\sigma}\right]-1}\right)=O\left(\frac{1}{n^{7 / 4}}\right), \quad \gamma_{n}^{\prime}(\infty)=0 .
\end{gathered}
$$

ДокАЗАТЕЛЬСтво. Имеем

$$
\gamma_{n}^{\prime}(t)=\frac{|c| J_{0}(\sqrt{t}|c|)}{2 \sqrt{t}\left(t-\lambda_{n}\right)}-\frac{J_{0}(\sqrt{t}|c|)}{\left(t-\lambda_{n}\right)^{2}}
$$

откуда непосредственной подстановкой, учитывая (4.19), получаем

$$
\begin{aligned}
& \gamma_{n}^{\prime}\left(\lambda_{n-\left[n^{\sigma}\right]-1}\right)-\gamma_{n}^{\prime}\left(\lambda_{n+\left[n^{\sigma}\right]+1}\right)=\frac{|c| J_{0}^{\prime}\left(\sqrt{\lambda_{n-\left[n^{\sigma}\right]-1}}|c|\right)}{2 \sqrt{\lambda_{n-\left[n^{\sigma}\right]-1}}\left(-2\left(\left[n^{\sigma}\right]+1\right)\right)} \\
& \quad-\frac{|c| J_{0}^{\prime}\left(\sqrt{\lambda_{n+\left[n^{\sigma}\right]+1}}|c|\right)}{2 \sqrt{\lambda_{n+\left[n^{\sigma}\right]+1}}\left(2\left(\left[n^{\sigma}\right]+1\right)\right)}+\frac{|c| J_{0}^{\prime}\left(\sqrt{\lambda_{n}}|c|\right)}{\sqrt{\lambda_{n}}\left(2\left(\left[n^{\sigma}\right]+1\right)\right)}+O\left(\frac{1}{n^{7 / 4-\sigma}}\right) .
\end{aligned}
$$

Из этой формулы в силу (4.18) немедленно следует оценка (4.22). Справедливость (4.23) следует из (4.24). Лемма 4.3 доказана.

Теперь, наконец, оценим последние два слагаемых в правой части (4.13). Имеет место 
Лемма 4.4. Пусть $0<\sigma<1 / 2$. Тогда при $n \gg 1 u(x, y) \in K(K-$ компакт в $\mathbb{R}^{2} \times \mathbb{R}^{2}$ )

$$
\begin{gathered}
\Gamma_{n}\left(\left[n^{\sigma}\right], n-\left[n^{\sigma}\right]-1\right)=O\left(\frac{1}{n^{3 \sigma}}\right), \\
\Gamma_{n}\left(n+\left[n^{\sigma}\right]+1, \infty\right)=O\left(\frac{1}{n^{3 \sigma}}\right) .
\end{gathered}
$$

ДокАЗАТЕЛЬСтво. Проверим оценку (4.26). Имеем

$$
\left|\Gamma_{n}\left(\left[n^{\sigma}\right], n-\left[n^{\sigma}\right]-1\right)\right| \leqslant \mathrm{const} \cdot \sum_{m=\left[n^{\sigma}\right]}^{n-\left[n^{\sigma}\right]-1} \max _{\lambda_{m-1} \leqslant t \leqslant \lambda_{m}}\left|\gamma_{n}^{\prime \prime \prime}(t)\right| .
$$

Используя неравенство (4.18) и определение функции $\gamma_{n}(t)$, получаем, что

$$
\begin{aligned}
\max _{\lambda_{m-1} \leqslant t \leqslant \lambda_{m}}\left|\gamma_{n}^{\prime \prime \prime}(t)\right| \leqslant & \frac{c_{1}}{\lambda_{m}^{7 / 4}\left|\lambda_{n}-\lambda_{m}\right|}+\frac{c_{2}}{\lambda_{m}^{5 / 4}\left|\lambda_{n}-\lambda_{m}\right|^{2}} \\
& +\frac{c_{3}}{\lambda_{m}^{3 / 4}\left|\lambda_{n}-\lambda_{m}\right|^{3}}+\frac{c_{4}}{\left|\lambda_{n}-\lambda_{m}\right|^{4}},
\end{aligned}
$$

где $c_{k}>0(k=\overline{1,4})$ и зависят только от $K$. Следовательно, справедливость оценки (4.26) вытекает из следующих соотношений:

$$
\begin{gathered}
\sum_{m=\left[n^{\sigma}\right]}^{n-\left[n^{\sigma}\right]-1} \frac{1}{\left(\lambda_{n}-\lambda_{m}\right) \lambda_{m}^{7 / 4}}=O\left(\frac{1}{n^{1+3 \sigma / 4}}\right), \\
\sum_{m=\left[n^{\sigma}\right]}^{n-\left[n^{\sigma}\right]-1} \frac{1}{\left(\lambda_{n}-\lambda_{m}\right)^{2} \lambda_{m}^{5 / 4}}=O\left(\frac{1}{n^{5 / 4+\sigma}}\right), \\
\sum_{m=\left[n^{\sigma}\right]}^{n-\left[n^{\sigma}\right]-1} \frac{1}{\left(\lambda_{n}-\lambda_{m}\right)^{3} \lambda_{m}^{3 / 4}}=O\left(\frac{1}{n^{3 / 4+2 \sigma}}\right), \\
\sum_{m=\left[n^{\sigma}\right]}^{n-1} \frac{1}{\left(\lambda_{n}-\lambda_{m}\right)^{4}}=O\left(\frac{1}{n^{3 \sigma}}\right) .
\end{gathered}
$$

Точно так же доказывается оценка (4.27). Лемма 4.4 доказана.

Теперь легко можем заключить, что доказательство теоремы 4.1 следует из формулы (4.13), оценок (4.14), (4.20), (4.21) и лемм 4.2-4.4. Теорема 4.1 доказана.

Исследуем функцию $\mathscr{B}_{n}^{(1,2)}(x, y)$. Из (4.7) получим, что

$$
\mathscr{B}_{n}^{(1,2)}(x, y)=\sum_{k=1}^{\left[n^{\sigma}\right]} \frac{J_{0}\left(\sqrt{\lambda_{n}+2 k}|c|\right)-J_{0}\left(\sqrt{\lambda_{n}-2 k}|c|\right)}{2 k} .
$$

Пусть $\sigma \in(0,1 / 2)$, тогда при $n \gg 1$, используя (4.17) и (4.19), приходим к следуюшему равенству

$$
\mathscr{B}_{n}^{(1,2)}(x, y)=\frac{|c| J_{0}^{\prime}\left(\sqrt{\lambda_{n}}|c|\right)}{2 \pi \sqrt{\lambda_{n}}}\left[n^{\sigma}\right]+O\left(\frac{1}{n^{7 / 4-3 \sigma}}\right) .
$$

Таким образом, из формул (4.6), (4.7), (4.30) и теоремы 4.1 заключаем, что для функции $\mathscr{B}_{n}^{(1)}(x, y)$ справедливо следуюшее утверждение. 
ТЕОРема 4.2. Пусть $0<\sigma<1 / 2$ и $K-$ произвольный компакт в $\mathbb{R}^{2} \times \mathbb{R}^{2}$. Тогда при $n \gg 1 u(x, y) \in K$

$$
\begin{aligned}
\mathscr{B}_{n}^{(1)}(x, y)=- & \frac{1}{4} Y_{0}\left(\sqrt{\lambda_{n}}|x-y|\right) \\
& +O\left(\frac{1}{n^{3 / 4}}\right)+O\left(\frac{1}{n^{7 / 4-3 \sigma}}\right)+O\left(\frac{1}{n^{1-\sigma}}\right)+O\left(\frac{1}{n^{3 \sigma}}\right) .
\end{aligned}
$$

Исследование асимптотического поведения функции $\mathscr{B}_{n}^{(2)}(x, y)$ проводится так же, как и функции $\mathscr{B}_{n}^{(1)}(x, y)$. Отметим, что сумма $(4.5)$ разбивается на суммы с четными и нечетными членами, к которым применяется лемма 0.1 об интегральном приближении. При этом главные члены асимптотики с четными и нечетными слагаемьми в $(4.5)$ взаимно уничтожаются. В итоге, для $\mathscr{B}_{n}^{(2)}(x, y)$ получим следуюшую асимптотику:

$$
\mathscr{B}_{n}^{(2)}(x, y)=O\left(\frac{1}{n^{3 / 4}}\right)+O\left(\frac{1}{n^{1-\sigma}}\right)+O\left(\frac{1}{n^{3 \sigma}}\right)+O\left(\frac{1}{n^{7 / 4-3 \sigma}}\right)
$$

где $0<\sigma<1 / 2$ и $(x, y) \in K\left(K-\right.$ компакт в $\left.\mathbb{R}^{2} \times \mathbb{R}^{2}\right)$.

Теперь из $(4.1),(4.4),(4.5)$ в силу леммы 4.1 , теоремы 4.2 и равенства $(4.32)$ легко получим асимптотическое представление ядра $\mathscr{B}_{n}(x, y)$. Для дальнейших наших целей достаточно, чтобы остаток в асимптотическом представлении был порядка $O\left(n^{-\lambda}\right), \lambda>1 / 2$, поэтому, полагая $\varepsilon=1 / 4$ в (4.3) и $\sigma=1 / 4$ в (4.2), (4.31), (4.32), приходим к основному результату этого параграфа:

Теорема 4.3. Пусть $K$ - произвольный компакт в $\mathbb{R}^{2} \times \mathbb{R}^{2}$. Тогда при $n \gg 1 u(x, y) \in K$

$$
\mathscr{B}_{n}(x, y)=-\frac{1}{4} Y_{0}\left(\sqrt{\lambda_{n}}|x-y|\right)+Q_{n}(x, y)
$$

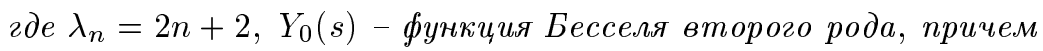

$$
Q_{n}(x, y)=O\left(\frac{\ln n}{n^{5 / 8}}\right)
$$

\section{§5. Асимптотика второй поправки теории возмущений}

В этом параграфе мы исследуем асимптотику чисел $\alpha_{n}=\operatorname{sp} P_{n} V B_{n} V P_{n}$ (см. (2.3)) при $n \gg 1$. А именно доказываем, что справедлива

TЕорема 5.1. Пусть $V \in C_{0}^{(4)}\left(\mathbb{R}^{2}\right)$, mогда при $n \gg 1$

$$
\alpha_{n}=O\left(n^{-\lambda}\right), \quad \lambda>1,
$$

т.е. последовательность $\alpha_{n}$ абсолютно суммируема. 
ДокАЗАТЕЛьство. Так как

$$
\alpha_{n}=\int_{\mathbb{R}^{2}} \int_{\mathbb{R}^{2}} V(x) V(y) \mathscr{B}_{n}(x, y) \mathscr{P}_{n}(x, y) d x d y,
$$

при $n \gg 1$ из (3.39) и теоремы 4.3 следует, что

$$
\alpha_{n}=\alpha_{n}^{(1)}+\alpha_{n}^{(2)}+\alpha_{n}^{(3)}+\alpha_{n}^{(4)}+\alpha_{n}^{(5)}+\alpha_{n}^{(6)},
$$

где

$$
\begin{aligned}
& \alpha_{n}^{(1)}=\frac{A_{0}}{8 \pi^{2}} \int_{\mathbb{R}^{2}} \int_{\mathbb{R}^{2}} V(x) V(y) J_{0}\left(\sqrt{\lambda_{n}}|x-y|\right) Y_{0}\left(\sqrt{\lambda_{n}}|x-y|\right) d x d y, \\
& \alpha_{n}^{(2)}=\frac{(-1)^{n} A_{0}}{8 \pi^{2}} \int_{\mathbb{R}^{2}} \int_{\mathbb{R}^{2}} V(x) V(y) J_{0}\left(\sqrt{\lambda_{n}}|x+y|\right) Y_{0}\left(\sqrt{\lambda_{n}}|x-y|\right) d x d y, \\
& \alpha_{n}^{(3)}=\frac{1}{2 \pi} \int_{\mathbb{R}^{2}} \int_{\mathbb{R}^{2}} V(x) V(y) J_{0}\left(\sqrt{\lambda_{n}}|x-y|\right) Q_{n}(x, y) d x d y, \\
& \alpha_{n}^{(4)}=\frac{(-1)^{n}}{2 \pi} \int_{\mathbb{R}^{2}} \int_{\mathbb{R}^{2}} V(x) V(y) J_{0}\left(\sqrt{\lambda_{n}}|x+y|\right) Q_{n}(x, y) d x d y, \\
& \alpha_{n}^{(5)}=\frac{A_{0}}{4 \pi} \int_{\mathbb{R}^{2}} \int_{\mathbb{R}^{2}} V(x) V(y) Y_{0}\left(\sqrt{\lambda_{n}}|x-y|\right) r_{n}(x, y) d x d y, \\
& \alpha_{n}^{(6)}=\int_{\mathbb{R}^{2}} \int_{\mathbb{R}^{2}} V(x) V(y) Q_{n}(x, y) r_{n}(x, y) d x d y, \quad A_{0}=-\pi .
\end{aligned}
$$

Положим в $(3.40) \sigma=1 / 4$, тогда из оценок (3.40) и теоремы 4.3 следует, что

$$
\alpha_{n}^{(6)}=O\left(n^{-\lambda}\right), \quad \lambda>1 .
$$

Далее, ядро резольвенты оператора $-\Delta$ в $\mathbb{R}^{2}[9 ;$ гл. $6, \S 2$, формула $(2.35)],[10 ;$ гл. 3, п. 3.7 , формула (8)]

$$
R(x, y, z)=\frac{i}{4} H_{0}(\sqrt{z}|x-y|)=\frac{i}{4}\left[J_{0}(\sqrt{z}|x-y|)+i Y_{0}(\sqrt{z}|x-y|)\right],
$$

и для того чтобы показать, что $\alpha_{n}^{(3)}, \alpha_{n}^{(4)}, \alpha_{n}^{(5)}$ есть величины порядка $O\left(n^{-\lambda}\right)$, $\lambda>1$, обратимся к следуюшей лемме работы $[11 ;$ п. 2 , лемма 3$]$ :

ЛЕмма 5.1. Пусть $f(|x|)$ - суммируемая положительная функиия такая, что $e^{-|x|} \leqslant f(|x|) \leqslant a_{1}|x|^{-1}, a_{1}>0$. Тогда для всех $z \neq 0$, в том числе $u z=\lambda \pm i 0$, оператор $F^{1 / 2} R(z) F^{1 / 2}$ ограничен $u\left\|F^{1 / 2} R(z) F^{1 / 2}\right\| \leqslant a|z|^{-1 / 2}$, $a>0$ не зависит от $z$, где $F$ - оператор умножсения на функиию $f(|x|), R(z)$ резольвента оператора $-\Delta$ в $\mathbb{R}^{2}$.

Теперь, используя эту лемму, оценим $\alpha_{n}^{(3)}$. Поскольку функция $V(x)$ финитна, воспользуясь равномерной на $K$ ( $K$ - компакт) оценкой (4.33) и неравенством Коши-Буняковского, получим

$$
\begin{aligned}
\left|\alpha_{n}^{(3)}\right| & =\left|\left(F^{1 / 2} \operatorname{Im} R\left(\lambda_{n}+i 0\right) F^{1 / 2} F^{-1 / 2} V Q_{n}, F^{-1 / 2} V\right)\right| \\
& \leqslant\left\|F^{1 / 2} R\left(\lambda_{n}+i 0\right) F^{1 / 2}\right\| \cdot\left\|F^{-1 / 2} V Q_{n}\right\| \cdot\left\|F^{-1 / 2} V\right\| \\
& \leqslant \frac{\text { const } \cdot\|V\|_{\infty}^{2} \ln n}{\lambda_{n}^{1 / 2} n^{5 / 8}} .
\end{aligned}
$$


Отсюда следует, что $\alpha_{n}^{(3)}=O\left(n^{-\lambda}\right), \lambda>1$. Такой же оценке удовлетворяют $\alpha_{n}^{(4)}, \alpha_{n}^{(5)}$. Отметим, что для оценки $\alpha_{n}^{(5)}$ достаточно воспользоваться (3.40) при $\sigma=1 / 4$.

Следовательно, теорема 5.1 будет доказана, если покажем, что $\alpha_{n}^{(1)}$ и $\alpha_{n}^{(2)}$ есть величины порядка $O\left(n^{-\lambda}\right), \lambda>1$. Справедлива

ЛЕмма 5.2. При $n \gg 1$

$$
\alpha_{n}^{(1)}=O\left(\frac{1}{n^{3 / 2}}\right)
$$

ДоКАЗАТЕЛЬСТво. Положим $y-x=s$ и $s=r e^{i \varphi}, 0 \leqslant \varphi \leqslant 2 \pi, 0 \leqslant r<\infty$, тогда из (5.2) получим, что

$$
\begin{aligned}
\alpha_{n}^{(1)}=\frac{A_{0}}{8 \pi^{2}} \int_{\mathbb{R}^{2}} V(x) \int_{0}^{\infty} \int_{0}^{2 \pi} V\left(x_{1}\right. & \left.+r \cos \varphi, x_{2}+r \sin \varphi\right) \\
\times & r J_{0}\left(\sqrt{\lambda_{n}} r\right) Y_{0}\left(\sqrt{\lambda_{n}} r\right) d r d \varphi d x
\end{aligned}
$$

Пусть $V \in C_{0}^{(2)}\left(\mathbb{R}^{2}\right)$ и $g\left(r, \lambda_{n}\right)=\int_{0}^{r} t J_{0}\left(\sqrt{\lambda_{n}} t\right) Y_{0}\left(\sqrt{\lambda_{n}} t\right) d t$, тогда, два раза интегрируя по частям в (5.2) по переменной $r$, имеем

$$
\alpha_{n}^{(1)}=\frac{A_{0}}{8 \pi^{2}} \lambda_{n}^{-3 / 2} \int_{0}^{\infty} \int_{0}^{\sqrt{\lambda_{n}} r} g(s) d s q(r) d r
$$

где

$$
\begin{aligned}
& q(r)= \int_{\mathbb{R}^{2}} V(x) \int_{0}^{2 \pi}\left[V_{1}^{\prime \prime}\left(x_{1}+r \cos \varphi, x_{2}+r \sin \varphi\right) \cos ^{2} \varphi\right. \\
&+V_{12}^{\prime \prime}\left(x_{1}+r \cos \varphi, x_{2}+r \sin \varphi\right) \sin 2 \varphi \\
&\left.+V_{2}^{\prime \prime}\left(x_{1}+r \cos \varphi, x_{2}+r \sin \varphi\right) \sin ^{2} \varphi\right] d \varphi d x, \\
& g(s)=\int_{0}^{s} t J_{0}(t) Y_{0}(t) d t
\end{aligned}
$$

причем [10; гл. 5, п. 5.12 , формула (2)]

$$
g(s)=\frac{s^{2}}{2}\left[Y_{0}(s) J_{0}(s)+J_{1}(s) Y_{1}(s)\right]
$$

Пусть $1 \ll M<N-$ фиксированное число, тогда интеграл (5.5) представим в следуюшем виде

$$
\begin{aligned}
\alpha_{n}^{(1)}= & \frac{A_{0}}{8 \pi^{2} \lambda_{n}^{3 / 2}}\left[\int_{0}^{N / \sqrt{\lambda_{n}}} \int_{0}^{\sqrt{\lambda_{n}} r} g(s) d s q(r) d r\right. \\
& \left.+\int_{N / \sqrt{\lambda_{n}}}^{\infty} \int_{0}^{M} g(s) d s q(r) d r+\int_{N / \sqrt{\lambda_{n}}}^{\infty} \int_{M}^{\sqrt{\lambda_{n}} r} g(s) d s q(r) d r\right] \\
= & \frac{A_{0}}{8 \pi^{2} \lambda_{n}^{3 / 2}}\left[I_{n}^{(1)}+I_{n}^{(2)}+I_{n}^{(3)}\right] .
\end{aligned}
$$


Отсюда будет следовать доказательство леммы 5.2 , если мы покажем, что $I_{n}^{(1)}$, $I_{n}^{(2)}, I_{n}^{(3)}$ есть величины порядка $O(1)$.

Действительно, так как $0 \leqslant s \leqslant N$ и $g(s)$ непрерьвна, а $q(r)$ ограничена и финитна, имеем

$$
\left|I_{n}^{(1)}\right| \leqslant \int_{0}^{N / \sqrt{\lambda_{n}}} \int_{0}^{\sqrt{\lambda_{n}} r}|g(s)| d s|q(r)| d r \leqslant \frac{c_{0} N^{2}}{\sqrt{\lambda_{n}}}, \quad\left|I_{n}^{(2)}\right| \leqslant c_{1},
$$

постоянная $c_{1}$ зависит только от $\operatorname{supp} V$ и $M$. Для оценки $I_{n}^{(3)}$, так как $s \geqslant M \gg 1$, из асимптотических формул для $Y_{i}(s), J_{i}(s)(i=0,1)[12 ;$ гл. $7, \S \S 4,5]$ вытекает, что

$$
g(s)=b_{0} \sin 2 s-\frac{b_{1}}{s} \cos 2 s+O\left(\frac{1}{s^{2}}\right) .
$$

Подставляя (5.8) в (5.7) и учитывая финитность $q(r)$, легко заключаем, что $I_{n}^{(3)}=O(1)$. Лемма 5.2 доказана.

Для исследования асимптотики $\alpha_{n}^{(2)}$ положим $y-x=s$ и перейдем к полярной системе координат: $s=(r, \varphi), x=(t, \psi)$. Тогда, применяя теорему сложения для цилиндрических функций $[6 ; \S 18$, формула $(3)]$, из $(5.2)$ получаем, что

$$
\begin{aligned}
\alpha_{n}^{(2)}= & B_{0} \sum_{k=-\infty}^{\infty} \int_{0}^{2 \pi} \int_{0}^{2 \pi} e^{i k(\varphi-\psi)} \int_{0}^{\infty} t J_{|k|}\left(2 \sqrt{\lambda_{n}} t\right) \int_{0}^{\infty} r J_{|k|}\left(\sqrt{\lambda_{n}} t\right) Y_{0}\left(\sqrt{\lambda_{n}} r\right) \\
& \times V\left(x_{1}, x_{2}\right) V\left(x_{1}+s_{1}, x_{2}+s_{2}\right) d r d t d \varphi d \psi
\end{aligned}
$$

здесь и в дальнейшем $x_{1}=t \cos \psi, x_{2}=t \sin \psi, s_{1}=r \cos \varphi, s_{2}=r \sin \varphi, B_{0}=$ $(-1)^{n} A_{0}\left(8 \pi^{2}\right)^{-1}$. Далее, $\alpha_{n}^{(2)}$ представим в виде суммы трех слагаемых. В первом слагаемом $-I_{n}: 0 \leqslant r \leqslant \lambda_{n}^{-1 / 2+\sigma}, t \in \mathbb{R}_{+}$, во втором $-J_{n}: \lambda_{n}^{-1 / 2+\sigma} \leqslant r<\infty$, $0 \leqslant t \leqslant 2^{-1} \lambda_{n}^{-1 / 2+\gamma}$, в третьем $-S_{n}: \lambda_{n}^{-1 / 2+\sigma} \leqslant r<\infty, 2^{-1} \lambda_{n}^{-1 / 2+\gamma} \leqslant t<\infty$, причем $\gamma, \sigma \in(0,1 / 2)$. Оценим каждое слагаемое.

Лемма 5.3. Пусть $V \in C_{0}^{(4)}\left(\mathbb{R}^{2}\right)$, тогда $п р и ~ n \gg 1$

$$
I_{n}=O\left(n^{-\lambda}\right), \quad \lambda>1 .
$$

ДокАЗАТЕЛЬСтво. Так как $r=|s| \in\left[0, \lambda_{n}^{-1 / 2+\sigma}\right]$ и $0<\sigma<1 / 2$, то

$$
V(x+s)=V(x)+\left[V_{1}^{\prime}(x) \cos \varphi+V_{2}^{\prime}(x) \sin \varphi\right] r+r^{2} Q(x, s, \theta),
$$

где

$$
Q(x, s, \theta)=\frac{1}{2}\left[V_{1}^{\prime \prime}(x+\theta s) \cos ^{2} \varphi+V_{12}^{\prime \prime}(x+\theta s) \sin 2 \varphi+V_{2}^{\prime \prime}(x+\theta s) \sin ^{2} \varphi\right]
$$

$\theta=\left(\theta_{1}, \theta_{2}\right), 0<\theta_{i}<1, i=1,2$. Далее, так как

$$
\int_{0}^{2 \pi} e^{i m \varphi} d \varphi= \begin{cases}2 \pi, & m=0 \\ 0, & m \neq 0\end{cases}
$$


подставляя (5.11) в (5.9), получаем, что

$$
\begin{aligned}
I_{n}= & B_{0} 2 \pi \int_{\mathbb{R}^{2}} V^{2}(x) J_{0}\left(2 \sqrt{\lambda_{n}}|x|\right) d x \int_{0}^{\lambda_{n}^{-1 / 2+\sigma}} r J_{0}\left(\sqrt{\lambda_{n}} r\right) Y_{0}\left(\sqrt{\lambda_{n}} r\right) d r \\
& +B_{0} 2 \pi \int_{0}^{\infty} \int_{0}^{2 \pi} V(x)\left[V_{1}^{\prime}(x) \cos \psi+V_{2}^{\prime}(x) \sin \psi\right] t J_{1}\left(2 \sqrt{\lambda_{n}} t\right) d t d \psi \\
& \times \int_{0}^{\lambda_{n}^{-1 / 2+\sigma}} r^{2} J_{1}\left(\sqrt{\lambda_{n}} r\right) Y_{0}\left(\sqrt{\lambda_{n}} r\right) d r \\
& +B_{0} \sum_{k=-\infty}^{\infty} \int_{0}^{\infty} \int_{0}^{2 \pi} t V(x) J_{|k|}\left(2 \sqrt{\lambda_{n}} t\right) \int_{0}^{\lambda_{n}^{-1 / 2+\sigma}} r^{3} J_{|k|}\left(\sqrt{\lambda_{n}} r\right) Y_{0}\left(\sqrt{\lambda_{n}} r\right) \\
& \times \int_{0}^{2 \pi} e^{i k(\varphi-\psi)} Q(x, s, \theta) d \varphi d \psi d r d t=I_{n}^{(1)}+I_{n}^{(2)}+I_{n}^{(3)} .
\end{aligned}
$$

Покажем, что

$$
\left|I_{n}^{(1)}\right| \leqslant \frac{\text { const }}{\lambda_{n}^{7 / 4}}
$$

Действительно,

$$
\left|\int_{0}^{\infty} t J_{0}\left(2 \sqrt{\lambda_{n}} t\right) q(t) d t\right|=\left|\int_{0}^{\infty} \psi_{n}(t) q^{\prime}(t) d t\right| \leqslant \frac{c_{1}}{\lambda_{n}^{3 / 4}}
$$

где

$$
\begin{aligned}
q(t) & =\int_{0}^{2 \pi} V^{2}(t \cos \psi, t \sin \psi) d \psi, \quad \psi_{n}(t)=\frac{1}{\lambda_{n}} \int_{0}^{\sqrt{\lambda_{n}} t} s J_{0}(2 s) d s \\
& \left|\int_{0}^{\lambda_{n}^{-1 / 2+\sigma}} r J_{0}\left(\sqrt{\lambda_{n}} r\right) Y_{0}\left(\sqrt{\lambda_{n}} r\right) d r\right|=\left|\frac{1}{\lambda_{n}} g\left(\lambda_{n}^{\sigma}\right)\right| \leqslant \frac{c_{2}}{\lambda_{n}},
\end{aligned}
$$

где функция $g(x)$ определяется формулой (5.6). Из (5.13) и (5.14) следует (5.12).

Теперь покажем, что

$$
\left|I_{n}^{(2)}\right| \leqslant \frac{\text { const }}{\lambda_{n}^{7 / 4-2 \sigma}}
$$

Из неравенства $(4.18)$ и $\left|\sqrt{s} Y_{0}(s)\right| \leqslant$ const при $V \in C_{0}^{(2)}\left(\mathbb{R}^{2}\right)$ легко следует, что

$$
\left|\int_{0}^{2 \pi} \int_{0}^{\infty} t J_{1}\left(2 \sqrt{\lambda_{n}} t\right) V(x)\left[V_{1}^{\prime}(x) \cos \psi+V_{2}^{\prime}(x) \sin \psi\right] d \psi d t\right| \leqslant \frac{c_{1}}{\lambda_{n}^{1 / 4}}
$$

и

$$
\left|\int_{0}^{\lambda_{n}^{-1 / 2+\sigma}} r^{2} J_{1}\left(\sqrt{\lambda_{n}} r\right) Y_{0}\left(\sqrt{\lambda_{n}} r\right) d r\right| \leqslant \frac{c_{2}}{\lambda_{n}^{3 / 2-2 \sigma}}
$$

откуда вытекает оценка (5.15). Наконец, если $V \in C_{0}^{(4)}\left(\mathbb{R}^{2}\right)$, то

$$
\int_{0}^{2 \pi} e^{i k \varphi} Q(x, \theta, r, \varphi) d \varphi=-k^{-2} \int_{0}^{2 \pi} e^{i k \varphi} Q_{\varphi}^{\prime \prime}(x, \theta, r, \varphi) d \varphi
$$


поэтому

$$
\left|I_{n}^{(3)}\right| \leqslant \frac{c_{3}}{\lambda_{n}^{9 / 4-3 \sigma}}
$$

Таким образом, оценка (5.10) следует из (5.12), (5.15) и (5.16) при $\sigma=1 / 4$. Поскольку переменные $t$ и $r$ входят в формулу (5.9) с одинаковым весом, учитьвая определения $I_{n}, J_{n}$, полагая $\gamma=1 / 4$, нетрудно доказать, что справедлива

ЛЕмма 5.4. Пусть $V \in C_{0}^{(4)}\left(\mathbb{R}^{2}\right)$, тогда при $n \gg 1$

$$
J_{n}=O\left(n^{-\lambda}\right), \quad \lambda>1
$$

Далее, имеет место

ЛЕмма 5.5. Пусть $V \in C_{0}^{(4)}\left(\mathbb{R}^{2}\right)$, тогда при $n \gg 1$

$$
S_{n}=O\left(n^{-\lambda}\right), \quad \lambda>1
$$

ДокАЗАТЕЛЬСТво. Пусть $1<\varepsilon<2$ и $\gamma=\sigma$, тогда положим

$$
\begin{aligned}
S_{n}= & \left(\sum_{|k| \leqslant \lambda_{n}^{\frac{\gamma}{1+\varepsilon}}}+\sum_{|k| \geqslant \lambda_{n}^{\frac{\gamma}{1+\varepsilon}}}\right) B_{0} \int_{0}^{2 \pi} \int_{0}^{2 \pi} e^{i k(\varphi-\psi)} \int_{\frac{1}{2} \lambda_{n}^{-1 / 2+\gamma}}^{\infty} t J_{|k|}\left(2 \sqrt{\lambda_{n}} t\right) \\
& \times \int_{\lambda_{n}^{-1 / 2+\gamma}}^{\infty} r J_{|k|}\left(\sqrt{\lambda_{n}} r\right) Y_{0}\left(\sqrt{\lambda_{n}} r\right) V(x) V(x+s) d \varphi d \psi d r d t \\
= & S_{n}^{(1)}+S_{n}^{(2)} .
\end{aligned}
$$

Оценим $S_{n}^{(1)}$. Так как $k \leqslant \lambda_{n}^{\frac{\gamma}{1+\varepsilon}}, 0<\gamma<1 / 2$ и $2 \sqrt{\lambda_{n}} t \geqslant \lambda_{n}^{\gamma}, \sqrt{\lambda_{n}} r \geqslant \lambda_{n}^{\gamma} \gg 1$, используя асимптотические формулы при $\varepsilon>1$ для функций $J_{k}\left(\sqrt{\lambda_{n}} r\right), J_{k}\left(2 \sqrt{\lambda_{n}} r\right)$ и $Y_{0}\left(\sqrt{\lambda_{n}} r\right)[12 ;$ гл. $7, \S \S 4,5]$, затем интегрируя по частям один раз по $t$ и дважды по $\varphi$, получаем, что

$$
\left|S_{n}^{(1)}\right| \leqslant \frac{\text { const }}{\lambda_{n}^{5 / 4}}
$$

Наконец, остается показать, что $S_{n}^{(2)}=O\left(n^{-\lambda}\right), \lambda>1$. Для этой цели в $(5.17)$ сделаем замену переменной $2 \sqrt{\lambda_{n}} t=\tau$, затем, используя равенство $\frac{d}{d \tau} \tau^{k+1} J_{k+1}(\tau)=$ $\tau^{k+1} J_{k}(\tau)$, интегрируем по частям по $\tau$. Тогда получим, что

$$
S_{n}^{(2)}=\sigma_{n}^{(1)}+\sigma_{n}^{(2)}+\sigma_{n}^{(3)}
$$


где

$$
\begin{aligned}
\sigma_{n}^{(1)}= & -\frac{B_{0}}{4 \lambda_{n}^{1-\gamma}} \sum_{|k| \geqslant \lambda_{n}^{1+\varepsilon}} \int_{0}^{2 \pi} \int_{0}^{2 \pi} e^{i k(\varphi-\psi)} \int_{\lambda_{n}^{-1 / 2+\gamma}}^{\infty} r J_{|k+1|}\left(\lambda_{n}^{\gamma}\right) J_{|k|}\left(\sqrt{\lambda_{n}} r\right) \\
& \times Y_{0}\left(\sqrt{\lambda_{n}} r\right) V\left(\frac{\cos \psi}{2 \lambda_{n}^{1 / 2-\gamma}}, \frac{\sin \psi}{2 \lambda_{n}^{1 / 2-\gamma}}\right) \\
& \times V\left(s_{1}+\frac{\cos \psi}{2 \lambda_{n}^{1 / 2-\gamma}, s_{2}}+\frac{\sin \psi}{2 \lambda_{n}^{1 / 2-\gamma}}\right) d \varphi d \psi d r, \\
\sigma_{n}^{(2)}= & \frac{B_{0}}{4 \lambda_{n}} \sum_{|k| \geqslant \lambda_{n}^{1+\varepsilon}} \int_{0}^{2 \pi} \int_{0}^{2 \pi} e^{i k(\varphi-\psi)} \int_{\lambda_{n}^{\gamma}}^{\infty} J_{|k+1|}(\tau) \int_{\lambda_{n}^{-1 / 2+\gamma}}^{\infty} r J_{|k|}\left(\sqrt{\lambda_{n}} r\right) \\
& \times Y_{0}\left(\sqrt{\lambda_{n}} r\right) V\left(x_{1}, x_{2}\right) V\left(s_{1}+x_{1}, s_{2}+x_{2}\right) d \tau d \varphi d \psi d r, \\
\sigma_{n}^{(3)}= & \frac{B_{0}}{4 \lambda_{n}^{3 / 2}} \sum_{|k| \geqslant \lambda_{n}^{1+\varepsilon}} \int_{0}^{2 \pi} \int_{0}^{2 \pi} e^{i k(\varphi-\psi)} \int_{\lambda_{n}^{\gamma}}^{\infty} \tau J_{|k+1|}(\tau) \int_{\lambda_{n}^{-1 / 2+\gamma}}^{\infty} r J_{|k|}\left(\sqrt{\lambda_{n}} r\right) \\
& \times Y_{0}\left(\sqrt{\lambda_{n}} r\right) \frac{\partial}{\partial t}\left(V\left(x_{1}, x_{2}\right) V\left(x_{1}+s_{1}, x_{2}+s_{2}\right)\right) d \tau d \varphi d \psi d r, \\
\tau= & 2 \sqrt{\lambda_{n}} t, \quad x_{1}=t \cos \psi, x_{2}=t \sin \psi, s_{1}=r \cos \varphi, s_{2}=r \sin \varphi .
\end{aligned}
$$

Из равномерных асимптотических формул для $J_{k}(k z)[12 ;$ гл. $11, \S 10$, формула (10.18)] следует, что для $k \gg 1$

$$
\left|J_{k}(\tau)\right| \leqslant \frac{\text { const }}{k^{1 / 2}}, \quad\left|J_{k}\left(\sqrt{\lambda_{n}} r\right)\right| \leqslant \frac{\text { const }}{k^{1 / 2}} .
$$

Следовательно, интегрируя по частям по переменной $\psi$ в (5.20) и используя (5.23), заключаем, что

$$
\left|\sigma_{n}^{(1)}\right| \leqslant \frac{\text { const }}{\lambda_{n}^{\frac{7}{4}-2 \gamma+\frac{\gamma}{1+\varepsilon}}}
$$

откуда при $\gamma=1 / 4$ для $n \gg 1$ имеем

$$
\sigma_{n}^{(1)}=O\left(n^{-\lambda}\right), \quad \lambda>1
$$

Далее, пусть $V \in C_{0}^{(4)}\left(\mathbb{R}^{2}\right)$, тогда, интегрируя четыре раза по частям в $(5.21)$, три раза по переменной $\varphi$ в (5.22) и используя неравенства $(5.23)$, получаем, что

$$
\left|\sigma_{n}^{(i)}\right| \leqslant \frac{\text { const }}{\lambda_{n}^{\frac{3}{4}+\frac{3 \gamma}{1+\varepsilon}}}, \quad i=2,3 .
$$

Отсюда при $\gamma=1 / 4$ и $\varepsilon<2$ следует, что для $n \gg 1$

$$
\sigma_{n}^{(i)}=O\left(n^{-\lambda}\right), \quad \lambda>1, \quad i=2,3 .
$$

Из (5.17)-(5.19), (5.24) и (5.25) вытекает утверждение леммы 5.5. А из лемм 5.3-5.5 следует, что $\alpha_{n}^{(2)}=O\left(n^{-\lambda}\right), \lambda>1$. Последнее утверждение вместе с леммой 5.2 завершают доказательство теоремы 5.1. 


\section{§6. Формула следов}

Из (2.2) и теорем 2.1 и 5.1 непосредственно следует, что ряд

$$
\sum_{n=0}^{\infty}\left[2(n+1)^{2}+\operatorname{sp} P_{n} V-\sum_{k=0}^{n} \mu_{k}^{(n)}\right]
$$

абсолютно сходится. Для вычисления суммы ряда (6.1) воспользуемся следующей леммой.

Лемма 6.1. Для любого $n$ имеет место тождество

$$
\begin{aligned}
\sum_{k=0}^{n} \alpha_{k}=\frac{1}{2 \lambda_{n}} & {\left[\operatorname{sp} E_{n} V^{2}-\sum_{k=0}^{n} \operatorname{sp}\left(P_{k} V\right)^{2}+2 \sum_{k=0}^{n} \lambda_{k} \alpha_{k}\right.} \\
& \left.+\sum_{k=0}^{n} \sum_{m=n+1}^{\infty}\left(\frac{2\left(\lambda_{n}-\lambda_{k}\right)}{\lambda_{m}-\lambda_{k}}-1\right) \operatorname{sp} P_{k} V P_{m} V\right]
\end{aligned}
$$

əде $E_{n}=\sum_{k=0}^{n} P_{k}$

ДокАЗАТЕЛьСтво. Из определения $\alpha_{k}($ см. (2.3)) имеем, что

$$
\alpha_{k}=\sum_{m \neq k}\left(\lambda_{m}-\lambda_{k}\right)^{-1} \operatorname{sp} P_{k} V P_{m} V P_{k}
$$

Пусть $\left\{\varphi_{s}^{(k)}\right\}_{k=0}^{\infty}, s=\overline{0, k},-$ ортонормированные собственные функции оператора $H_{0}$, тогда

$$
a_{m k}=\operatorname{sp} P_{k} V P_{m} V P_{k}=\sum_{s=0}^{k} \sum_{l=0}^{m}\left|\left(V \varphi_{s}^{(k)}, \varphi_{l}^{(m)}\right)\right|^{2}
$$

Заметим, что $a_{m k}=a_{k m}$, следовательно, $\sum_{k=0}^{n} \sum_{m=0, m \neq k}^{n}\left(\lambda_{m}-\lambda_{k}\right)^{-1} a_{m k}=0$. Учитывая это равенство, имеем

$$
\begin{aligned}
2 \lambda_{n} \sum_{k=0}^{n} \alpha_{k} & =2 \lambda_{n} \sum_{k=0}^{n} \sum_{m=n+1}^{\infty} \frac{a_{m k}}{\lambda_{m}-\lambda_{k}}-2 \sum_{k=0}^{n} \lambda_{k} \alpha_{k}+2 \sum_{k=0}^{n} \lambda_{k} \alpha_{k} \\
& =-2 \sum_{k=0}^{n} \sum_{\substack{m=0 \\
m \neq k}}^{n} \frac{\lambda_{k} a_{m k}}{\lambda_{m}-\lambda_{k}}+2 \sum_{k=0}^{n} \sum_{m=n+1}^{\infty} \frac{\lambda_{n}-\lambda_{k}}{\lambda_{m}-\lambda_{k}} a_{m k}+2 \sum_{k=0}^{n} \lambda_{k} \alpha_{k}
\end{aligned}
$$

Теперь преобразуем первое слагаемое в (6.4). Легко заметить, что

$$
\sum_{k=0}^{n} \sum_{\substack{m=0 \\ m \neq k}}^{n} \frac{\lambda_{k} a_{m k}}{\lambda_{m}-\lambda_{k}}=-\sum_{k=0}^{n} \sum_{\substack{m=0 \\ m \neq k}}^{n} a_{m k}-\sum_{k=0}^{n} \sum_{\substack{m=0 \\ m \neq k}}^{n} \frac{\lambda_{m} a_{m k}}{\lambda_{k}-\lambda_{m}}
$$

откуда, так как $a_{m k}=a_{k m}$, следует равенство

$$
-2 \sum_{k=0}^{n} \sum_{\substack{m=0 \\ m \neq k}}^{n} \frac{\lambda_{k} a_{m k}}{\lambda_{m}-\lambda_{k}}=\sum_{k=0}^{n} \sum_{\substack{m=0 \\ m \neq k}}^{n} a_{m k}
$$


Далее, учитывая (6.3) и пользуясь равенством Парсеваля, получаем, что

$$
\begin{aligned}
\sum_{k=0}^{n} \sum_{\substack{m=0 \\
m \neq k}}^{n} a_{m k} & =\sum_{k=0}^{n} \sum_{m=0}^{n} a_{m k}-\sum_{k=0}^{n} a_{k k} \\
& =\sum_{k=0}^{n}\left[\operatorname{sp}\left(P_{k} V^{2}\right)-\operatorname{sp}\left(P_{k} V\right)^{2}\right]-\sum_{k=0}^{n} \sum_{m=n+1}^{\infty} a_{m k}
\end{aligned}
$$

Из этого равенства, если учесть (6.4) и (6.5), получим тождество (6.2). Лемма 6.1 доказана.

Отметим, что сумма ряда (6.1) равна пределу при $n \rightarrow \infty$ левой части (6.2). Для вычисления этого предела изучаем поведение при $n \rightarrow \infty$ величин, стоящих в правой части (6.2).

Так как $\lambda_{k}=2 k+2$, из теоремы 5.1 вытекает, что при $n \rightarrow \infty$

$$
\sum_{n=0}^{n} \lambda_{k} \alpha_{k}=o(n)
$$

Справедлива

ЛЕмма 6.2. При $n \rightarrow \infty$

$$
\sum_{k=0}^{n} \sum_{m=n+1}^{\infty}\left(\frac{2\left(\lambda_{n}-\lambda_{k}\right)}{\lambda_{m}-\lambda_{k}}-1\right) \operatorname{sp} P_{k} V P_{m} V=o(n)
$$

ДоказАтельство. Действительно, так как $k \leqslant n<m$ и $V \varphi_{s}^{(k)} \in L^{2}\left(\mathbb{R}^{2}\right)$, $s=\overline{0, k}$, то в силу равенства Парсеваля

$$
\sum_{m=n+1}^{\infty}\left|\operatorname{sp} P_{k} V P_{m} V\right|=o(1)
$$

откуда следует (6.7). Лемма 6.2 доказана.

ЛЕмма 6.3. При $n \rightarrow \infty$

$$
\sum_{k=0}^{n} \operatorname{sp}\left(P_{k} V\right)^{2}=o(n)
$$


ДоКАЗАТЕЛЬСТво. Пусть $0<\gamma<1$ и

$$
\sum_{k=0}^{n} \operatorname{sp}\left(P_{k} V\right)^{2}=\sum_{k=0}^{\left[n^{\gamma}\right]} \operatorname{sp}\left(P_{k} V\right)^{2}+\sum_{k=\left[n^{\gamma}\right]+1}^{n} \operatorname{sp}\left(P_{k} V\right)^{2}
$$

Тогда так как

$$
\operatorname{sp}\left(P_{k} V\right)^{2}=\int_{\mathbb{R}^{2}} \int_{\mathbb{R}^{2}} V(x) V(y) \mathscr{P}_{k}^{2}(x, y) d x d y
$$

и в силу глобальной оценки $(0.4)$ для $(x, y) \in K\left(K-\right.$ компакт в $\left.\mathbb{R}^{2} \times \mathbb{R}^{2}\right)\left|\mathscr{P}_{k}(x, y)\right| \leqslant$ const, при $n \rightarrow \infty$ имеем

$$
\sum_{k=0}^{\left[n^{\gamma}\right]} \operatorname{sp}\left(P_{k} V\right)^{2}=o(n)
$$

Для оценки второго слагаемого в правой части (6.8), поскольку $k \geqslant\left[n^{\gamma}\right] \gg 1$, воспользуемся теоремой 3.2, откуда в силу (6.9) получаем

$$
\begin{aligned}
\operatorname{sp}\left(P_{k} V\right)^{2}= & \int_{\mathbb{R}^{2}} \int_{\mathbb{R}^{2}} V(x) V(y) J_{0}^{2}\left(\sqrt{\lambda_{k}}|x-y|\right) d x d y \\
& +\int_{\mathbb{R}^{2}} \int_{\mathbb{R}^{2}} V(x) V(y) J_{0}^{2}\left(\sqrt{\lambda_{k}}|x+y|\right) d x d y \\
& +\int_{\mathbb{R}^{2}} \int_{\mathbb{R}^{2}} V(x) V(y) J_{0}\left(\sqrt{\lambda_{k}}|x-y|\right) J_{0}\left(\sqrt{\lambda_{k}}|x+y|\right) d x d y \\
& +O\left(\frac{1}{k^{1 / 2+\sigma / 2}}\right)+O\left(\frac{1}{k^{1-3 \sigma / 2}}\right) .
\end{aligned}
$$

Покажем, что для $k \gg 1$

$$
\operatorname{sp}\left(P_{k} V\right)^{2}=O\left(k^{-1 / 2}\right) .
$$

С этой целью сначала произведем замену переменной в правой части (6.11), полагая $x-y=2 s, x+y=2 \tau$, и переходя к полярной системе координат, получаем, например, для первого слагаемого оценку

$$
\begin{aligned}
\int_{\mathbb{R}^{2}} & \int_{\mathbb{R}^{2}} V(x) V(y) J_{0}^{2}\left(\sqrt{\lambda_{k}}|x-y|\right) d x d y \\
= & 2 \int_{\mathbb{R}^{2}} d \tau \int_{0}^{2 \pi} \int_{0}^{\infty} V\left(\tau_{1}+r \cos \varphi, \tau_{2}+r \sin \varphi\right) \\
& \times V\left(\tau_{1}-r \cos \varphi, \tau_{2}-r \sin \varphi\right) r J_{0}^{2}\left(2 \sqrt{\lambda_{k}} r\right) d r d \varphi=O\left(\frac{1}{\sqrt{\lambda_{k}}}\right) .
\end{aligned}
$$

Здесь мы воспользовались финитностью $V$ и неравенством (4.18). Аналогично получим, что второе и третье слагаемые в (6.11) имеют порядок $O\left(k^{-1 / 2}\right)$. Следовательно, если положить $0<\sigma<1 / 3$ для четвертого слагаемого, то оценка (6.12) доказана. Из (6.8), (6.10) и (6.12) следует лемма 6.3.

Таким образом, из (6.6) и лемм 6.2 и 6.3, так как $\lambda_{n}=2 n+2$, следует, что пределы при $n \rightarrow \infty$ последних трех слагаемых в правой части (6.2) равны нулю. Для вычисления предела первого слагаемого докажем следующую теорему. 
Tеорема 6.1. Пусть $W$ - оператор умножения на функиию $W(x)$ из класса $C_{0}^{(4)}\left(\mathbb{R}^{2}\right)$. Тогда при $n \rightarrow \infty$ справедлива асимптотическая формула

$$
\operatorname{sp} E_{n} W=\frac{n+1}{2 \pi} \int_{\mathbb{R}^{2}} W(x) d x+\frac{1}{4 \pi} \int_{\mathbb{R}^{2}}\left(1-x^{2}\right) W(x) d x+o(1),
$$

где $x^{2}=x_{1}^{2}+x_{2}^{2}$.

ДокАЗАТЕЛЬСтво. Пусть $p_{l}^{(i)}$ - проектор на одномерное собственное подпространство, соответствующее собственному значению $2 l+1$ оператора $-\frac{d^{2}}{d x_{i}}+x_{i}^{2}$, $i=1,2$. Тогда $P_{m}=\sum_{l=0}^{m} p_{l}^{(1)} \otimes p_{m-l}^{(2)}$ и

$$
E_{n}=\sum_{m=0}^{n} \sum_{k=0}^{m} p_{l}^{(1)} \otimes p_{m-l}^{(2)}=\sum_{l=0}^{n} p_{l}^{(1)} \otimes \sum_{s=0}^{n-l} p_{s}^{(2)} .
$$

Пусть $0<\delta<1$, тогда после несложных преобразований из (6.14) получим

$$
\begin{aligned}
E_{n}= & E_{n}^{(1)}+E_{n}^{(2)}-\frac{1}{2}\left[e_{\left[n^{\delta}\right]}^{(1)} \otimes e_{n-\left[n^{\delta}\right]-1}^{(2)}+e_{\left[n^{\delta}\right]}^{(2)} \otimes e_{n-\left[n^{\delta}\right]-1}^{(1)}\right] \\
& +\frac{1}{2}\left[\sum_{l=\left[n^{\delta}\right]+1}^{n-\left[n^{\delta}\right]-1} p_{l}^{(1)} \otimes e_{n-l}^{(2)}+\sum_{l=\left[n^{\delta}\right]+1}^{n-\left[n^{\delta}\right]-1} p_{l}^{(2)} \otimes e_{n-l}^{(1)}\right],
\end{aligned}
$$

где

$$
e_{m}^{(i)}=\sum_{s=0}^{m} p_{s}^{(i)}, \quad E_{n}^{(1)}=\sum_{s=0}^{\left[n^{\delta}\right]} p_{s}^{(1)} \otimes e_{n-s}^{(2)}, \quad E_{n}^{(2)}=\sum_{s=0}^{\left[n^{\delta}\right]} p_{s}^{(2)} \otimes e_{n-s}^{(1)} .
$$

Обозначим через $E_{n}(x, y), p_{n}\left(x_{i}, y_{i}\right), e_{n}\left(x_{i}, y_{i}\right), i=1,2$, соответственно ядра операторов $E_{n}, p_{n}^{(i)}, e_{n}^{(i)}, i=1,2$. Так как

$$
\operatorname{sp} E_{n} W=\int_{\mathbb{R}^{2}} E_{n}(x, x) W(x) d x
$$

в силу представления (6.15) нам необходимо изучить асимптотику ядер $p_{n}\left(x_{i}, x_{i}\right)$ и $e_{n}\left(x_{i}, x_{i}\right), i=1,2$. Из асимптотических формул (0.5) следует, что при $n \rightarrow \infty$

$$
p_{n}\left(x_{i}, x_{i}\right)=f_{n}^{2}\left(x_{i}\right)=\widetilde{p}_{n}\left(x_{i}, x_{i}\right)+\widehat{p}_{n}\left(x_{i}, x_{i}\right),
$$

где

$$
\begin{gathered}
\widetilde{p}_{n}\left(x_{i}, x_{i}\right)=\frac{1}{\pi}\left[\frac{1}{\sqrt{2 n+1}}+\frac{a_{1}\left(x_{i}\right)}{(2 n+1)^{3 / 2}}+\frac{a_{2}\left(x_{i}\right)}{(2 n+1)^{5 / 2}}+O\left(\frac{1}{n^{7 / 2}}\right)\right], \\
a_{1}\left(x_{i}\right)=\frac{x_{i}^{2}}{2}, \quad a_{2}\left(x_{i}\right)=\frac{3}{8} x_{i}^{4}-\frac{1}{16}, \\
\widehat{p}_{n}\left(x_{i}, x_{i}\right)=\frac{1}{\pi} \cos \left[2 \sqrt{2 n+1} x_{i}-\pi n\right] \cdot\left[\frac{1}{\sqrt{2 n+1}}+O\left(\frac{1}{n^{3 / 2}}\right)\right] \\
+\frac{1}{\pi} \sin \left[2 \sqrt{2 n+1} x_{i}-\pi n\right] \cdot\left[\frac{x_{i}^{3}}{3(2 n+1)}+O\left(\frac{1}{n^{2}}\right)\right] .
\end{gathered}
$$


Далее, из формулы Дарбу-Кристоффеля [7; гл. 3, п. 3.2, формула (3.2.4)] следует, что

$$
e_{n}\left(x_{i}, x_{i}\right)=\sum_{k=0}^{n} p_{k}\left(x_{i}, x_{i}\right)=\sqrt{\frac{n+1}{2}}\left[f_{n+1}^{\prime}\left(x_{i}\right) f_{n}\left(x_{i}\right)-f_{n+1}\left(x_{i}\right) f_{n}^{\prime}\left(x_{i}\right)\right]
$$

откуда, используя (0.5), при $n \gg 1$ получаем

$$
e_{n}\left(x_{i}, x_{i}\right)=\widetilde{e}_{n}\left(x_{i}, x_{i}\right)+\widehat{e}_{n}\left(x_{i}, x_{i}\right)
$$

где

$$
\begin{aligned}
\widetilde{e}_{n}\left(x_{i}, x_{i}\right)= & \frac{1}{\pi}\left[\sqrt{2 n+1}+\frac{b_{1}\left(x_{i}\right)}{\sqrt{2 n+1}}+\frac{b_{2}\left(x_{i}\right)}{(2 n+1)^{3 / 2}}+O\left(\frac{1}{n^{5 / 2}}\right)\right] \\
& b_{1}\left(x_{i}\right)=\frac{1-x_{i}^{2}}{2}, \quad b_{2}\left(x_{i}\right)=-\frac{1}{16}+\frac{x_{i}^{2}}{4}-\frac{x_{i}^{4}}{8} \\
\widehat{e}_{n}\left(x_{i}, x_{i}\right)= & \frac{(-1)^{n}}{2 \pi}\left\{\cos (\sqrt{2 n+1}+\sqrt{2 n+3}) x_{i} \cdot\left[-\frac{1}{\sqrt{2 n+1}}+O\left(\frac{1}{n^{3 / 2}}\right)\right]\right. \\
& \left.+\sin (\sqrt{2 n+1}+\sqrt{2 n+3}) x_{i} \cdot\left[-\frac{x_{i}^{3}}{3(2 n+1)}+O\left(\frac{1}{n^{2}}\right)\right]\right\} .
\end{aligned}
$$

Теперь из формул (6.16), (6.20), (6.21), неравенства (0.4) при $0<\delta<1 / 3$ легко заключаем, что для $n \gg 1$

$$
\begin{gathered}
E_{n}^{(i)}(x, x)=\frac{1}{\pi} \sqrt{2 n+1} \cdot e_{\left[n^{\delta}\right]}\left(x_{i}, x_{i}\right)+o(1), \quad i=1,2 \\
e_{n-\left[n^{\delta}\right]-1}\left(x_{i}, x_{i}\right) \cdot e_{\left[n^{\delta}\right]}\left(x_{j}, x_{j}\right)=\frac{1}{\pi} \sqrt{2 n+1} \cdot e_{\left[n^{\delta}\right]}\left(x_{j}, x_{j}\right)+o(1), \quad i, j=1,2 .
\end{gathered}
$$

Отсюда в силу (6.15), (6.20) и (6.21) получаем, что при $1 / 5<\delta<1 / 3$ для $n \gg 1$

$$
\begin{aligned}
E_{n}^{(1)}(x, x) & +E_{n}^{(2)}(x, x) \\
-\frac{1}{2}[ & \left.e_{n-\left[n^{\delta}\right]-1}\left(x_{1}, x_{1}\right) e_{\left[n^{\delta}\right]}\left(x_{2}, x_{2}\right)+e_{n-\left[n^{\delta}\right]-1}\left(x_{2}, x_{2}\right) e_{\left[n^{\delta}\right]}\left(x_{1}, x_{1}\right)\right] \\
= & \frac{1}{\pi^{2}} \sqrt{2 n+1}\left[\sqrt{2\left[n^{\delta}\right]+1}+\frac{b_{1}\left(x_{1}\right)+b_{1}\left(x_{2}\right)}{2 \sqrt{2\left[n^{\delta}\right]+1}}+\frac{b_{2}\left(x_{1}\right)+b_{2}\left(x_{2}\right)}{2\left(2\left[n^{\delta}\right]+1\right)^{3 / 2}}\right] \\
& \quad+\frac{1}{2 \pi} \sqrt{2 n+1}\left[\widehat{e}_{\left[n^{\delta}\right]}\left(x_{1}, x_{1}\right)+\widehat{e}_{\left[n^{\delta}\right]}\left(x_{2}, x_{2}\right)\right]+o(1) .
\end{aligned}
$$

Наконец, изучим асимптотическое поведение ядра последнего слагаемого в правой части равенства (6.15). А именно, используя асимптотические формулы 
(6.18)-(6.21), при $1 / 5<\delta<1 / 3$ для $n \gg 1$ получаем, что

$$
\begin{aligned}
& \sum_{l=\left[n^{\delta}\right]+1}^{n-\left[n^{\delta}\right]-1} p_{l}\left(x_{1}, x_{1}\right) e_{n-l}\left(x_{2}, x_{2}\right) \\
& \quad=\frac{1}{\pi^{2}} \sum_{l=\left[n^{\delta}\right]+1}^{n-\left[n^{\delta}\right]-1}\left\{\frac{\sqrt{2(n-l)+1}}{\sqrt{2 l+1}}+a_{1}\left(x_{1}\right) \frac{\sqrt{2(n-l)+1}}{(2 l+1)^{3 / 2}}\right. \\
& \quad+a_{2}\left(x_{1}\right) \frac{\sqrt{2(n-l)+1}}{(2 l+1)^{5 / 2}}+\frac{b_{1}\left(x_{2}\right)}{\sqrt{2 l+1} \sqrt{2(n-l)+1}} \\
& \left.\quad+O(\sqrt{2(n-l)+1}) \pi \widehat{p}_{l}\left(x_{1}, x_{1}\right)+O\left(\frac{1}{\sqrt{2 l+1}}\right) \pi \widehat{e}_{n-l}\left(x_{2}, x_{2}\right)\right\}+o(1) .
\end{aligned}
$$

Применяя лемму 0.1 об интегральном приближении к суммам в (6.23), при $1 / 5<$ $\delta<1 / 3$ получаем

$$
\begin{aligned}
& \sum_{l=\left[n^{\delta}\right]+1}^{n-\left[n^{\delta}\right]-1} \frac{\sqrt{2(n-l)+1}}{\sqrt{2 l+1}}=\frac{\pi}{2}(n+1)-\sqrt{(2 n+1)\left(2\left[n^{\delta}\right]+1\right)} \\
& \quad-\frac{1}{2} \sqrt{\frac{2 n+1}{2\left[n^{\delta}\right]+1}}+\frac{\sqrt{2 n+1}}{12\left(2\left[n^{\delta}\right]+1\right)^{3 / 2}}+o(1), \\
& \sum_{l=\left[n^{\delta}\right]+1}^{n-\left[n^{\delta}\right]-1} \frac{\sqrt{2(n-l)+1}}{(2 l+1)^{3 / 2}}=-\frac{\pi}{2}+\sqrt{\frac{2 n+1}{2\left[n^{\delta}\right]-1}}-\frac{\sqrt{2 n+1}}{2\left(2\left[n^{\delta}\right]+1\right)^{3 / 2}}+o(1), \\
& \sum_{l=\left[n^{\delta}\right]+1}^{n-\left[n^{\delta}\right]-1} \frac{\sqrt{2(n-l)+1}}{(2 l+1)^{5 / 2}}=\frac{1}{3} \frac{\sqrt{2 n+1}}{2\left(2\left[n^{\delta}\right]+1\right)^{3 / 2}}+o(1), \\
& \sum_{l=\left[n^{\delta}\right]-1} \frac{1}{\sqrt{2 l+1} \sqrt{2(n-l)+1}}=\frac{\pi}{2}+o(1) .
\end{aligned}
$$

Далее, подставив (6.24) в (6.23), затем (6.23) и (6.19) в аналогичное (6.15) равенство для ядра $E_{n}(x, x)$, при $1 / 5<\delta<1 / 3$ получим

$$
\begin{aligned}
E_{n}(x, x)= & \frac{1}{2 \pi}(n+1)+\frac{1}{4 \pi}\left(1-x_{1}^{2}-x_{2}^{2}\right)+\frac{\sqrt{2 n+1}}{2 \pi}\left[\widehat{e}_{\left[n^{\delta}\right]}\left(x_{1}, x_{1}\right)+\widehat{e}_{\left[n^{\delta}\right]}\left(x_{2}, x_{2}\right)\right] \\
& +\frac{1}{\pi} \sum_{l=\left[n^{\delta}\right]+1}^{n-\left[n^{\delta}\right]-1}\left\{O(\sqrt{2(n-l)+1})\left[\widehat{p}_{l}\left(x_{1}, x_{1}\right)+\widehat{p}_{l}\left(x_{2}, x_{2}\right)\right]\right. \\
& \left.+O\left(\frac{1}{\sqrt{2 l+1}}\right)\left[\widehat{e}_{n-l}\left(x_{1}, x_{1}\right)+\widehat{e}_{n-l}\left(x_{2}, x_{2}\right)\right]\right\}+o(1)
\end{aligned}
$$


Подставляя (6.25) в (6.17), учитывая асимптотические формулы для $\widehat{p}_{n}\left(x_{i}, x_{i}\right)$ и $\widehat{e}_{n}\left(x_{i}, x_{i}\right)$ из $(6.19)$ и $(6.21)$, интегрируя четыре раза по частям $\left(W(x) \in C_{0}^{(4)}\left(\mathbb{R}^{2}\right)\right)$, при $1 / 5<\delta<1 / 3$ получаем формулу (6.13). Теорема 6.1 доказана.

Теперь из теоремы 6.1, лемм 6.2 и 6.3 , оценки (6.6) в силу равенства (6.2) следует, что имеет место следующая теорема.

Tеорема 6.2. Пусть $V \in C_{0}^{(4)}\left(\mathbb{R}^{2}\right)$. Тогда

$$
\sum_{n=0}^{\infty}\left[2(n+1)^{2}+\operatorname{sp} P_{n} V-\sum_{k=0}^{n} \mu_{k}^{(n)}\right]=\frac{1}{8 \pi} \int_{\mathbb{R}^{2}} V^{2}(x) d x
$$

Теоремы 6.1 и 6.2 составляют основу для получения классической формулы первого регуляризованного следа оператора $H$. Из теоремы 6.2 вытекает, что при $N \gg 1$

$$
\sum_{n=0}^{N}\left[2(n+1)^{2}+\operatorname{sp} P_{n} V-\sum_{k=0}^{n} \mu_{k}^{(n)}\right]=\frac{1}{8 \pi} \int_{\mathbb{R}^{2}} V^{2}(x) d x+o(1),
$$

следовательно, так как $\sum_{n=0}^{N} \operatorname{sp} P_{n} V=\operatorname{sp} E_{N} V$, учитывая (6.13), имеем

$$
\sum_{n=0}^{N}\left[2(n+1)^{2}+C_{0}-\sum_{k=0}^{n} \mu_{k}^{(n)}\right]=C_{1}+o(1)
$$

где

$$
C_{0}=\frac{1}{2 \pi} \int_{\mathbb{R}^{2}} V(x) d x, \quad C_{1}=\frac{1}{4 \pi} \int_{\mathbb{R}^{2}}\left(x^{2}-1\right) V(x) d x+\frac{1}{8 \pi} \int_{\mathbb{R}^{2}} V^{2}(x) d x .
$$

Таким образом, мы приходим к следуюшему основному утверждению о первом регуляризованном следе оператора $H$ :

Teоpema 6.3. Пусть $V \in C_{0}^{(4)}\left(\mathbb{R}^{2}\right)$. Тогда

$$
\sum_{n=0}^{\infty}\left[2(n+1)^{2}+C_{0}-\sum_{k=0}^{n} \mu_{k}^{(n)}\right]=\frac{1}{8 \pi} \int_{\mathbb{R}^{2}} V^{2}(x) d x-\frac{C_{0}}{2}+\frac{1}{4 \pi} \int_{\mathbb{R}^{2}} x^{2} V(x) d x
$$

где $C_{0}=\frac{1}{2 \pi} \int_{\mathbb{R}^{2}} V(x) d x, x^{2}=x_{1}^{2}+x_{2}^{2}, \mu_{k}^{(n)}$ - собственнье значения оператоpa $H, k=\overline{0, n}$. 


\section{Список литературы}

1. Гельфанд И. М., Левитан Б. М. Об одном простом тождестве для собственных значений дифференциального оператора второго порядка // Докл. АН СССР. 1953. Т. 88. C. $593-596$.

2. Лидский В. Б., Садовничий $B . A$. Регуляризованные суммы корней одного класса целых функций // Функц. анализ и его прилож. 1967. Т. 1. № 2. С. 52-59.

3. Садовничий В. А., Дубровский В. В. Классическая формула регуляризованного следа для собственных чисел оператора Лапласа-Бельтрами с потенциалом на сфере // Докл. AH CCCP. 1991. T. 319. № 1. C. 61-62.

4. Podolskii V. E. On summability of regularized sums of eigenvalues of the Laplace-Beltrami operator with potential on symmetric spaces of rank one // Russian J. Math. Phys. 1996. V. 3. № 4. P. 1-8.

5. Любишкин В. А. Регуляризованньй след двумерного гармонического осциллятора // Матем. заметки. 1993. Т. 53. №3. С. 156-158.

6. Никифоров А.Ф., Уваров В.Б. Специалњные функции математической физики. М.: Наука, 1984.

7. Сегё Г. Ортогоналњные многочлены. М.: ГИФМЛ, 1962.

8. Като Т. Теория возмущений линейных операторов. М.: Мир, 1972.

9. Березанский Ю. М. Разложения по собственньм функциям самосопряженных операторов. Киев: Наукова думка, 1965.

10. Ватсон Г. Н. Теория бесселевых функций. М.: ИЛ, 1949.

11. Муртазин X. X., Садовничий B. А. Спектральньй анализ многочастичного оператора Шрёдингера. М.: Изд-во МГУ, 1988.

12. Олвер Ф. Асимптотика и специальные функции. М.: Наука, 1990.

Башкирский государственный университет, г. Уфа Поступила в редакцию 12.05 .1999 и 14.12 .2000 